\section{Cerebrovascular}

Diseases

Cerebrovasc Dis 2004; $18: 352-378$

DOI: $10.1159 / 000081968$

The abstracts are only available online, free of charge, under www.karger.com/doi/10.1159/000081968

\title{
ISS Regional Cerebrovascular Diseases Conference
}

Beijing, China, September 23-28, 2004

\section{Selected Abstracts}

Cochaired by

Julien Bogousslavsky, Lausanne, Switzerland

Xinfeng Liu, Nanjing, China

Yongjun Wang, Beijing, China 
Cerebrovascular Diseases
Cerebrovasc Dis 2004; $18: 352-378$

DOI: $\underline{10.1159 / 000081968}$

\section{Effects of Carotid Stenting and Angioplasty in Treating Carotid Bifurcation Stenosis - Reports from Chinese Neurologists}

X. Liu, Q. Yin, R. Zhang, G. Xu

Department of Neurology, Jinling Hospital, Medical

College of Nanjing University, Nanjing, China

Background: Carotid artery stenting and angioplasty (CAS) is becoming increasingly popular among various specialties for treating primary and recurrent carotid artery stenosis. The morbidity and mortality associated with this procedure is improving but the immediate and long-term results remains relatively unclear especially in developing countries. This study investigated the initial results of carotid artery angioplasty with stenting performed by neurologists for treating symptomatic patients with carotid bifurcation occlusion in a general hospital located in southeast China. Patients who had a history of stroke, transient ischemic attack (TIA) or amaurosis fugax in previous 6 months were selected for CAS if they had carotid bifurcation stenosis more then $60 \%$ (diameter) in degree confirmed by carotid angiography. Methods: Since November 2003, 56 carotid arteries in 44 patients underwent CAS, with distal filter embolization protection used in all patients. Mean patient age was 59.4 years; $77.3 \%$ of patients were men. Indications for CAS included transient ischemic attack (TIA; 45.5\%), stroke (50.1\%), and amaurosis fugax (4.5\%). Mean internal carotid artery diameter stenosis was $79.2 \%$. Pathologic conditions were simple stenosis $(63.6 \%)$, recurrent carotid stenosis with plaque $(27.3 \%)$, and stenosis with ulcer $(9.1 \%)$. In $36.4 \%$ of cases the contralateral carotid artery had $80 \%$ or greater stenosis or was completely occluded. Results: Technical success was achieved in $100 \%$ of cases. There were no deaths, no major strokes, one minor stroke (National Institutes of Health Stroke Scale, 3), and two TIA (neurologic event rate, 9.1\%). The single minor stroke resolved completely by 1 week. Two patient $(9.1 \%)$ had a prolonged hypotension relieved within 2 weeks. No patient had perioperative myocardial infarction. Transient neurologic changes occurred in 2 patients $(9.1 \%)$ during pre- and after-dilatation and all resolved with deflation. Bradyarrhythmia requiring pharmacologic treatment occurred in 3 patients (13.6\%). At mean follow-up of 4 months there has been no recurrent stenosis as noted by duplex scanning and carotid angiography. Conclusions: CAS with cerebral protection can be performed safely in symptomatic Chinese patients of carotid artery stenosis, with low perioperative morbidity and mortality. The effectiveness and safety of the procedure must be determined with longer follow-up.

\section{The Nanjing Stroke Registry Program: Founding of a Hospital-based Retrospective and Prospective Stroke Registry in China}

\author{
G. Xu, X. Liu, O. Yin, R. Zhang, G. Zhou \\ Department of Neurology, Jinling Hospital, Medical \\ College of Nanjing University, Nanjing, China
}

Background and Purpose: The epidemiologic and clinical data of stroke concerning Chinese population is very lacking. This program aims to assess the mortality, incidence, case-fatality rate and risk factors of stroke as well as the effects of prophylactic and therapeutic interventions in a community-based Chinese population. Methods: A hospital-based stroke registry has been established in the year of 2002 in Nanjing City which located in southeast China. The Nanjing city has about 3,500,000 inhabitants and is geographically somewhat isolated and has a low emigration-immigration rate. More than ninetynine percent of the dwellers are Han Chinese, and the population has an age-sex distribution like that of Mainland China as a whole. All patients falling into one of nine diagnostic categories of stroke or transient ischemic attack are registered on admission to a general hospital. The medical, social, and demographic data are abstracted onto coding data forms. A small group of neurologists assign definitive diagnoses according to standardized criteria after reviewing all of the medical data. The pervious 10 years stroke patients were also screened retrospectively by studying the medical history recording. Results: The stroke registry provides a new and powerful tool for collecting population-based data on a large number of cases in a relatively shorttime. After adjusting for demographic differences, epidemiologic studies can be carried out that may be generalized to the entire Chinese population. Conclusions: The completeness of the stroke ascertainment and the large population registered also offer an excellent opportunity for any interested researcher to investigate the relationships between medical, social, and demographic conditions on the one hand and stroke risk on the other; to study the efficacy of prevention and treatment programs; and to determine health care provision requirements in Han Chinese population.

\section{Mild Cognitive Impairment may Prodrome Vascular Dementia as Dementia of Alzheimer's Type}

\author{
X. Fan, G. Xu, X. Liu, R. Zhang, Q. Yin \\ Department of Neurology, Jinling Hospital, Medical \\ College of Nanjing University, Nanjing, China
}

Background and Purpose: It is now well accepted that mild cognitive impairment (MCI) is a prodromal stage preceding dementia

\begin{tabular}{ll}
\hline KARGER & (c) 2004 S. Karger AG, Basel \\
1015-9770/04/0184-0352\$21.00/0 \\
$\begin{array}{l}\text { Fax +4161306 1234 } \\
\text { E-Mail karger@karger.ch } \\
\text { www.karger.com }\end{array}$ & $\begin{array}{l}\text { Accessible online at: } \\
\text { www.karger.com/ced }\end{array}$
\end{tabular}


of Alzheimer's type (DAT). Vascular dementia (VaD), at least some types, shares similar risk factors with DAT and may mimic its clinical course. Weather MCI present as a prodrome for $\mathrm{VaD}$ as well as to DAT is still controversial. This study aims to seek out if there is a relationship between MCI and $\mathrm{VaD}$ in elderly people. Methods: Subjects were from an outpatient department of a general hospital. Serial cognitive tests, Mini-Mental State Examination and Cognitive Capacity Screening Examination, were administered at 3-6 monthly intervals for each subject. Neuroimaging scan (CT or MRI) was performed annually. Subtle cognitive impairment was weighted according to established MCI criteria. Subjects identified with MCI were then followed for an additional $3.45 \pm 3.23$ years. Diagnosis of DAT was made according to NINCDS/ADRDA criteria and of VaD according to NINCDS/AIRENS criteria. Results: During $3.05 \pm 2.37$ years of MCI follow up, 79 (23.9\%) of 330 cognitively normal subjects developed MCI. 29 subjects developed $\mathrm{VaD}$, of which, 17 $(58.6 \%)$ had prodromal MCI. Of these, two thirds were sub-classified as small vessel dementia. The remaining $12(41.4 \%)$ of $\mathrm{VaD}$ were diagnosed directly from a cognitively normal status without preceding MCI. These were predominantly multi-infarct or strategic-infarct dementia (69.7\%). An additional $36(48.0 \%)$ of MCI subjects developed DAT. Both VaD and DAT diagnosed after MCI prodromes manifested similar spectral domains of cognitive impairments, including memory, during their MCI stages. Conclusions: In some VaD subtypes, particularly those caused by subcortical microvascular disease or multiple lacuna infarctions, dementia may be preceded by MCI. These kinds of dementia may have similar domains of cognitive impairment and similar progressive course to that of DAT.

\section{Intranasal Delivery of Inductive Agents to Rats Leads to In Vivo Proliferation and Differentiating of Adult Neural Progenitor Cells in Subventricular Zone in Rats}

\author{
H. Ma, X. Liu, R. Guo, R. Zhang, W. Wu, X. Mao \\ Department of Neurology, Jinling Hospital, Medical \\ College of Nanjing University, Nanjing, China
}

Objective: Basic fibroblast growth factor is a polypeptide not only with potent multi-potential trophic effects but also stem cell inducing effects. This study was carried out to investigate the distribution of bFGF by several administration methods and the general pharmacological effects of bFGF on proliferation and differentiating of adult neural progenitor cells in vivo in sub-ventricular zone. Methods: Firstly the bFGF was delivered to the rats by several in vivo methods including intravenous administration, intranasal delivery, the radio-immunity methods, immunohistochemistry technique and Western blotting methods were used to detect the distribution of the delivered agents; then mature SD rats received, with or without, intranasal administration of bFGF, previously or immediately after focal ischemia infarcts were made in the right lateral cerebral cortex by permanent distal middle cerebral artery occlusion. Intraoperative measurements of core temperature, arterial blood pressure and blood geses, blood glucose concentration, and hematocrit and postoperative measurements of temperature revealed no differences among bFGFtreated and MCAO animals. 1, 4, 7, 14 days later, animals were killed 8.5-10 hours after BrdU injection. Results: Using several different in vivo assays, we found that the bFGF concentrations in olfactory bulb and sub-ventricular zone are higher by intranasal than intravenous. And using anti-BrdU and different special stem cell antibodys by the immno-fluorescence double-staining methods we assess that the intranasal group showed more double-stained neural progenitor cells than the intravenous group; also we found the several migration stem cells from the sub-ventricular zone. Conclusions: Our study showed that intranasal bFGF in vivo could induce the proliferation and differentiating and even migration of the adult progenitor cells in adult sub-ventricular zone.

\section{The Effect and Mechanism of HS706 on the Metabolism of Arachidonic Acid in Rats Following Cerebral Ischemia and Reperfusion}

\author{
R. Zhang, O. Dong, X. Liu, H. Ren, C. Lv \\ Department of Neurology, Jinling Hospital, Medical \\ College of Nanjing University, Nanjing, China
}

Background: It was demonstrated that the flavanoids, such as ginkgo biloba, quercetin and baicalein, notably protect brain tissue from the ischemic insults through inhibiting the synthesis of oxygen free radical, prostaglandins and leukotrienes. Previously, we observed that HS706 $\left(\mathrm{C}_{21} \mathrm{H}_{18} \mathrm{O}_{11}\right)$, a kind of flavanoid, inhibited the inflammatory reaction and reduced the infarct volume after the cerebral ischemic-reperfusion. Purpose: To discuss the protective mechanisms of HS706 in rats following focal cerebral ischemia and reperfusion. Methods: 18 male SD rats were selected and randomly divided into HS706-treating, control and sham operated group $(n=6)$. The model of middle cerebral artery occlusion and recanalization was performed using a standard intraluminal procedure as previously described. RT-PCR and Western blot were used to detect the expression of cyclooxygenase-2 (COX-2) and 5-lipoxygenase (5-LOX), and their products prostaglandin $\mathrm{E}_{2}\left(\mathrm{PGE}_{2}\right)$ and cysteine-containing leukotrienes (cys-LTs) were evaluated with EIA respectively. The change of albumin in the brain tissue was detected by SDS-PAGE. Results: HS706 inhibited the synthesis of cys-LTs $(54.736 \pm 9.564 \mathrm{pg} / \mathrm{g}$ vs $124.899 \pm 15.957 \mathrm{pg} / \mathrm{g}, \mathrm{P}<0.01)$ by the means of down-regulation of the cytosolic 5-LOX. The concentration of albumin in the brain homogenate was decreased in rats treated with HS706 (1.271 \pm 0.116 vs $1.645 \pm 0.206, \mathrm{P}<0.01)$. HS706 might attenuate the mRNA expression of COX-2 after cerebral ischemia-reperfusion $(0.515 \pm$ 0.048 vs $0.661 \pm 0.080, \mathrm{P}<0.05)$. Conclusions: HS706 could decrease the translocation of 5-LOX, and reduce the synthesis of cys-LTs. Another, HS706 could reduce the level of albumin in the brain tissue and inhibit the induced expression of COX-2 at the mRNA level. 


\section{Macromolecular Transport Across Blood- Brain Barrier During Pathologic Conditions}

\section{R. Guo, G. Xu, X. Liu}

Department of Nurology, Jinling Hospital, Medical College of Nanjing University, Nanjing, China

The blood-brain barrier (BBB) is a diffusion barrier, which impedes influx of most compounds from blood to brain under physiologic conditions. Because macromolecular are large, highly polarized molecules, their rate of transporting across the BBB is low. Under some pathologic conditions, a number of chemical mediators are released that increase BBB permeability, such as brain tumor, Alzheimer disease, multiple sclerosis and stroke. We reviewed here the recent developments in our understanding of the role of the BBB dysfunctionin CNS disease and there is an increased permeability to some macromolecular. Though different macromolecular according to different disease, there are apparent structural similarities in the mechanisms of transporting them across CNS microvessels. These CNS conduits become patent in damaged endothelial cells (ECs) for the passage of macromolecules, and purportedly for inflammatory and neoplastic cells as well. EC structures were observed in association with increased BBB permeability of tracers including exogenously injected HRP, normally excluded from the intercellular milieu of the CNS. Subsequent studies of non-BBB-type tumor ECs determined that others defined the EC VTS and other vesicular structures as vesiculo-vacuolar organelles. Finally, this review describes nanoparticulate systems for brain delivery of macromolecular. Overcoating with some special materials seems to lead to the adsorption of apolipoprotein $\mathrm{E}$ from blood plasma onto the nanoparticle surface. The particles then seem to mimic low-density lipoprotein (LDL) particles and could interact with the LDL receptor leading to their uptake by the endothelial cells.

\section{Expanding the Therapeutic Window for Edaravone (MCl-186) Coadministered with t-PA in a Rat Model of Embolic Stroke}

\author{
W. Wu, X. Liu, F. Shao, G. Xu \\ Department of Nurology, Jinling Hospital, \\ Medical College of Nanjing University, Nanjing, China
}

Background and Purpose: Thrombolysis with t-PA beyond 3-hour therapeutic window fails to provide therapeutic benefit. Preclinical studies suggested that ischemia-reperfusion initiated by delayed thrombolysis could precipitate further brain injury. Many of the pathophysiologic events triggered by reperfusion are mediated through the production of free radicals. Edaravone (MCI-186) is a potent scavenger of hydroxyl radicals. We tested the hypothesis that combination treatment of Edaravone and t-PA expand the therapeutic window for ischemic stroke. Methods: The middle cerebral artery (MCA) of male wistar rats were occluded by a single fibrin-rich clot. After embolization, rats were randomized into the following groups: t-PA treatment group, in which t-PA was intravenously administered at 3 or 6 hours after MCA occlusion; Edaravone treatment group, in which edaravone was intravenously administered at the dosage of
$5 \mathrm{mg} / \mathrm{kg}$ after MCA occlusion; combination treatment group, in which edaravone was intravenously administered in combination with t-PA 3 or 6 hours after MCA occlusion; Control group, in which equal volume of saline was given at 3 or 6 hours after MCA occlusion. Neurological deficit was evaluated at 24 hours after MCA occlusion. Tissue sections were then analyzed for lesion volume, $\mathrm{Bcl}_{2}$ expression and TUNEL staining. Results: A significant $(\mathrm{P}<0.05)$ improvement in neurologic recovery and a significant $(\mathrm{P}<0.05)$ reduction in lesion volume were observed in combination treatment groups and t-PA treatment groups compared with controls. No increase in hemorrhagic transformation was observed in combination treatment groups. The number of $\mathrm{Bcl}_{2}$-positive cells was significantly $(\mathrm{P}<0.05)$ increased in the combination treatment groups compared with controls. The number of TUNEL-positives cells was significantly $(\mathrm{P}<0.05)$ decreased in the combination treatment groups compared with controls. Conclusions: This study suggests that the combination treatment of Edaravone and t-PA expands the therapeutic window for ischemic stroke to at least 6 hours after embolization.

\section{Monitoring Migration and Differentiation of Murine Embryonic Stem Cells After Implantation into Rat Olfactory Bulb}

\author{
X. Jian, X. Gelin, L. Xinfeng \\ Neurology Department of Jinling Hospital, Medical \\ College of Nanjing University, Nanjing, China
}

Neural stem cells or progenitor cells are known to be born continually in the subventricular zone (SVZ) in the forebrain and migrate to the olfactory bulb (OB) along the rostral migratory stream (RMS), a specific pathway. We attempted in this study to confirm the pathway from the opposite direction and to detect the differentiation pattern of the implanted embryonic stem cells (ESCs) on rats suffering from permanent middle cerebral artery occlusion (MCAO). Embryonic stem cells were labeled via transduction procedure with lentiviral vector containing enhanced green fluorescent protein (GFP) together with a MRI contrast agent. Then the ESCs stably expressing GFP in vitro culture were stereotactically implanted into the olfactory bulb of rats 24 hours after permanent MCAO and healthy rats respectively. The implanted cells were observed in vivo by MRI with high contrast against the host tissue and were confirmed by GFP registration later. Neuronal markers of $\mathrm{NeU}$ and MAP2 were detected by fluorescent immunohistochemistry. Now this study is still ongoing and we expected to find out the possible migration of the implanted cells along the RMS targeting other parts of the brain like SVZ or cerebral lesion area. And also we want to test if the implanted cells can differentiate to functional neurons or neural progenitor cells by immunohistochemical results. A trend of difference between the MACO rats and the healthy control was found in pre-study. Affirmative outcome of this study may raise a possibility to find some noninvasive way for stem cell implantation. 


\section{Neuroprotection of Quercetin on Neuronal Apoptosis Following Neuronal Injury}

\author{
Y. Zhu, N. Jin, J. Zhou, X. Liu, G. Xu \\ Department of Neurology, Jinling Hospital, \\ Medical College of Nanjing University, Nanjing, China
}

Background: Quercetin, a natural flavonoid found in many fruits, vegetables and herbs, has been reported having potential stroke proventive and antithrombotic functions. Epidemiological and molecular biological researches have confirmed that quercetin can protect neurons against apoptosis and necrosis following neuronal injury. Methods: After the simultaneous incubation of PC12 cells with $200 \mu \mathrm{mol} / \mathrm{L}$ hydrogen peroxide $\left(\mathrm{H}_{2} \mathrm{O}_{2}\right)$ and quercetin $(0,5,10,15$ and $20 \mu \mathrm{mol} / \mathrm{L}$ ) for $30 \mathrm{~min}$, we used MTT assay, flow cytometrical analysis and Western blotting to evaluate the neuroprotective effects of quercetin on injured PC12 cells. Results: Results from MTT assay showed that quercetin increased PC12 cells survival rate in a dosedependent manner. Compared with control, 10-20 $\mu \mathrm{mol} / \mathrm{L}$ quercetin significantly decreased apoptosis rate of PC12 cells $(1.65, \mathrm{p}<0.01)$. Results from Western blotting showed that quercetin obviously upregulated the expression of $\mathrm{p} 53(1.34, \mathrm{p}<0.05)$ and $\mathrm{Bcl}-2(1.76$, $\mathrm{p}<0.01)$ protein of injured PC12 cells. Conclusions: These results suggest that quercetin can protect neuron from apoptosis following neuronal injury. This neuroprotective function is probably related to the up-regulation of $\mathrm{p} 53$ and $\mathrm{Bcl}-2$ expressions.

\section{Effect of Urokinase on Expression of Platelet Glycoprotein Ilb/Illa in Patients with Acute Brain Infarction}

\author{
M. He, L. Cao, H. Zhao \\ Department of Neurology, First Affiliated Hospital, \\ Peking University, Beijing, China
}

Objective: To assess platelet activation after urokinase thrombolysis in patients with acute brain infarction. Methods: Expression of platelet glycoprotein IIb/IIIa (GP IIb/IIIa) were serially evaluated on admission and 1 hour, 6 hours, 24 hours after intravenous thrombolysis with 1.5 million units of UK over 30 min in 15 patients with acute brain infarction which treated within 6 hours of symptom onset by whole blood flow cytometry, and compared to the measurements in the healthy control subjects and the routine-treatment subjects. Results: The patients with acute brain infarction showed highly significant increases in the percentage of GPIIb/IIIa-positive circulating platelets and the mean fluorescene intensity at the baseline and the follow-up levels, compared with the healthy subjects. But there were no differences between pre-treatment and post treatment in routinetreatment subjects. Platelet activation was inhibited slightly after thrombolytic therapy in most of the cases. The attenuated platelet activation reached significance 6 hours after thrombolysis compared with the baselines. But no significant differences could be detected between the baselines and the assessments 1 hour or 24 hours after thrombolysis. Platelet activation was enhanced after thrombolytic therapy in some cases, especially 3 patients with reinfarction syndrome. Conclusions: Platelets can be activated or inhibited during
UK thrombolysis process. Individual assessment of antiplatelet treatment during thrombolysis is desirable to prevent infarct-related artery reocclusion or intracranial hemorrhage.

\section{Basic Fibroblast Growth Factor Can Improve the Treatment Effect of Cerebral Infarction}

\author{
X. Li, C. Yao \\ Biopharmaceutical Research \& Development Center, \\ Jinan University, Guangzhou, China
}

Background and Objective: Basic Fibroblast Growth Factor, bFGF, is neurotrophic factor with multifunctional bioactivity. It can improve vascular formation and microcirculation, accelerate the development and growth of central and peripheral nerve system, and protect the apoptosis of nerve cells. In vitro researches showed that bFGF had potential usefulness as a protection and treatment for stroke. In this study, we would evaluate the therapeutic effectiveness of bFGF on cerebral infarction. Methods: 90 patients with cerebral infarction were divided two groups, control group (40 patients) and bFGF treatment group (50 patients). The ages, disease times, case history and concomitant diseases of two group patients had not significantly difference $(P>0.05)$. The control patients were treated with conventional methods, such as expanding the blood vessel, dissolving throbus, controlling the blood pressure, decreasing the intracranial pressure, reducing the blood viscidity and anti-infective etc. The other patients were treated conventional methods, and in addition with bFGF. 4000 IU bFGF was injected intramuscularly every day and continuously treated for 20 days. Results: There were 30 and 46 effective cases in the control group and the bFGF treatment group respectively, and the total effective rate was $75 \%$ and $92 \%$ of the control group and the bFGF treatment group respectively $(P<0.05)$. The defect index of nerve function of the control group and the bFGF treatment group was $22.33 \pm 8.31$ and $21.20 \pm 8.96$ respectively before treatment $(P>0.05)$, and it was decreased to $14.38 \pm 8.61$ and $11.10 \pm 6.02$ respectively after treatment $(P<0.05)$. Conclusions: bFGF can improve the therapeutic effectiveness of cerebral infarction with conventional methods, decrease the disability and incidence rate of sequela, and raise patients' life quality.

\section{Cerebral Arteriopathy in CADASIL Patients \\ W. Zhang, H. Kalimo, G. Zhu \\ Department of Neurology, Beijing General Hospital of PLA, Beijing, China}

Objective: In cerebral autosomal dominant arteriopathy with subcortical infarcts and leukoencephalopathy (CADASIL) arteriolar walls are thickened with destruction of vascular smooth muscle cells (VSMCs) and deposits of granular osmiophilic material (GOM). Small infarcts occur in the white matter (WM) and deep grey matter (GM) while the cerebral cortex is sparing. We analysed the arteriolar structural changes in CADASIL patients. Materials and Methods: The sclerotic index (SI) of arterioles in GM and WM was analysed in 
four patients with CADASIL caused by C475T (R133C) mutation in Notch 3 and in six age-matched controls. We also reconstructed 9 arterioles from 1,000 serial sections of penetrating arterioles or their branches in two CADASIL patients. Results: SI of CADASIL group was significantly higher than that of control group in both GM and WM. In CADASIL group SI was significantly higher in WM than in GM. Distribution curve of the arteriolar internal diameters in CADASIL shifted towards smaller sizes than those in the controls. In serial sections, SI of WM penetrating arterioles or their branches did not increase sharply until the internal diameters had decreased to about $20-30 \mu \mathrm{m}$ and external diameters to about $100-130 \mu \mathrm{m}$. Conclusions: In CADASIL long penetrating arterioles or branches supplying subcortical structures are obliterated and their walls are thickened. Our present study supports the findings of reduction in cerebral blood flow (CBF) or regional cerebral blood volume (rCBV) in WM of CADASIL and conforms to clinical abundance of infarcts and primary ischemic damage in CADASIL patients.

\section{Endovascular Thrombolysis and Stent Angioplasty for Occlusion in Cerebral Venous Sinuses}

\author{
B. Li, M. Guo, S. Li, M. Wang \\ Department of Interventional Radiology, \\ General Hospital of PLA, Beijing, China
}

Objective: Using direct thromblysis and stent angioplasty to treat the occlusion of cerebral venous sinuses in 17 cases were reported here. Methods: All of 17 cases with the thrombosis and stenosis in venous sinuses were confirmed by DSA (Digital substract angiography). There were 3 cases with thrombosis in single sinus and 14 cases with thrombosis in multiple sinuses. The circulating period was prolonged for over 13 seconds. The micro-catheter was preserved in sinus for 3 to 5 days and infused urokinase one million IU and oral wafarin $3-5 \mathrm{mg}$ each day during treatment. The stent angioplasty was done for 4 cases with obvious venous sinus stenosis that were showed by DSA after post-thrombolysis. Results: After contact thrombolysis and stent angioplasty in sinuses among 17 cases, remarkable recanalization of occllusive sinuses compared with before in $9 / 17$ cases and partial in $8 / 17$ cases were achieved. After treatment, the ICP was returned to normal in 7/17 cases, $8 / 17$ cases with relapse of thrombosis in different degree after 7 days kept for good state via the continued anticoagulation. There were only 2 cases with the ICP above $280 \mathrm{mmHg}$. No obvious relapse in $15 / 17$ cases were found follow-up 3-60 months. Conclusions: The primary results of our group demonstrated that the successive thrombolysis and stent angioplasty for occlusion and thrombosis of venous sinuses are the effective and safe therapies which directly promoting drainage for cerebral venous blood and rapidly decreasing the ICP.

\section{A New Modified Classification of Spinal Vascular Malformations}

\section{F. Ling}

Department of Neurosurgery, Xuanwu Hospital, Beijing, China

Objective: To propose a new modified classification of spinal vascular malformations. Methods: Since 1986 to March 2003, we had treated 549 cases of spinal vascular malformations. The imaging and clinical data were reviewed. The anatomy, angioarchitecture and pathophysiology were carefully analyzed. The previous classification, we have published in 1996, was modified. Results: The new classification includes all spinal vascular lesions in our group. Conclusions: The new modified classification offers several advantages: 1) it includes all the spinal vascular lesions, and nomenclatural terms are definite. 2) It emphasizes the target of treatment in its category. 3) It reveals the characters of spinal vascular malformations, based on neuroanatomy, pathophysiology, angiographic and intraoperative observation.

\section{Stent-assisted Angioplasty of Intracranial Vertebrobasilar Artery Stenosis}

\author{
J. Liu, B. Hong, Y. Xu \\ Department of Neurosurgery, Changhai Hospital, \\ Second Military Medical University, Shanghai, China
}

Objective: To evaluate the feasibility, safety, and efficacy of endovascular stent-assisted angioplasty for the treatment of vertebrobasilar artery stenosis, and to discuss our preliminary experience and perioperative management. Methods: Fourty-two symptomatic patients with 43 intracranial vertebrobasilar stenoses presented with recurrent transient ischemic attacks or with history of infarction of posterior circulation territory were treated with balloon-expanded stents. The locations of stenoses were intracranial vertebral artery in 30 and basilar artery in 13 . The average stenosis rate was $(76.4 \pm 10.3) \%$ $(50 \%-90 \%)$ and the length of the stenosis was $(6.7 \pm 3.2) \mathrm{mm}$ of average $(3-12 \mathrm{~mm})$. Results: The technique was successful for Fourtyone cases with 42 stenoses without procedural and perioperative death or major stroke. One minor stroke $(2.4 \%)$ occurred in one patient of combined vertebral and basilar stenoses during operation. The average residual stenosis after stenting was $(10.3 \pm 5.4) \%$. There was no death or cerebral ischemic attach for all patients who were clinical followed for 6 to 40 months after stenting. Three restenosis was noted for the 15 patients who were angiographically followed for an average of 8.5 months after treatment. Conclusions: Endovascular stenting for vertebrobasilar artery stenosis is effective and safe. But the long-term results need further investigation. 


\section{Analysis of 1353 Consecutive Patients in Chengdu Stroke Register}

M. Liu

Stroke Clinical Research Unit, Department of Neurology, West China Hospital, Sichuan University, Chengdu, China

Background and Purpose: stroke register study can provide useful data for better understanding on clinical patterns, risk factors and outcomes in patients with stroke. It can provide planning data for clinical trials in the treatment of stroke and is also a critical tool to document the status of stroke care and to help improvements in the quality of stroke care. Few prospective consecutive stroke register study was conducted in China. It is difficult to know accurately the features of Chinese stroke patients and compare data with other populations. This study set up a Chengdu Stroke Register and analyzed the first 2 year's data, in order to obtain reliable clinical information of Chinese stroke patients. Methods: From March 1, 2002 to Feb 29, 2004, we prospectively registered stroke patients consecutively admitted to Department of Neurology, West China Hospital, Sichuan University in Chengdu, China. The baseline demographic, risk factors, treatment, and outcome data was recorded. We followed up patients at one, three, six months and one year for death, disability and recurrence. Results: a total of 1,353 consecutive patients were registered during the first 2 years. Male: $58 \%$, female: $42 \%$. Age ranged from 10 to 98 years old. The average age was $62.6 \pm 14.4$ years old. $93 \%$ of patients completed CT/MRI scanning. $11 \%$ of patients arrived in hospital within 3 hours of stroke onset and $21 \%$ within 6 hours. Ischemic stroke account for $60 \%$, Intracranial hemorrhage for $29 \%$, subarachnoid hemorrhage $6 \%$, TIA $4 \%$, and unknown type for $1 \%$. Death or disability was $40 \%$ at one month, $31 \%$ at 3 month. Other data is in analyzing. Conclusions: reliable clinical data of Chinese stroke patients in hospital setting can be obtained by prospectively registering patients consecutively admitted hospital. Chengdu Stroke Register was established and will publish its serial results in future.

\section{The Correlative Study on Glucose Metabolism of the Brain Using ${ }^{18}$ F-FDG PET and Cognition in Patients with Vasculer Dementia}

\section{Wang, J. Zhao, X. Zhou \\ Department of Neurology, Guangdong Provincial People's Hospital, Guangzhou, China}

Objective: To study the correlation between the images of 18 F-FDG PET of the brain and cognition in Patients with Vasculer Dementia (VD). Methods: 13 patients with VD and 13 age, sex and education matched normal controls (NC) were studied. 18F-FDG PET brain scan was performed in all the patients and controls, Wechsler Adult Intelligence Scale-Revised in China (WAIS-RC), Mini Mental Status Examination (MMSE) was adopted to examine all the patients and controls. 13 VD patients and 13 controls were examined by the magnetic resonance imaging (MRI). Results: $100 \%$ of PD patients showed abnormal PET images. The Rcl/cb of cerebral lobes and basal ganglion in VD patients was significantly decreased than in NC. There were correlation between the extent of cognitive disturbance and the degree and range of decreasing of glucose metabolism in Vasculer Dementia. The metabolism decreased almost on the local of cerebral lobes in mild cases of VD patients, but it might be decreased either on the cortical and subcortical of cerebral lobs in serious cases of VD. The scores of PIQ, VIQ, FIQ and MMSE in VD patients were less then that in the normal controls, $P<0.01$. VIQ was less than PIQ in VD patients which more decreasing of glucose metabolism in cortical but PIQ was less then VIQ in which more decreasing of glucose metabolism in subcortical and basal ganglion. Conclusions: VD patients had cognitive disturbance. It might be correlation to the degree and range of cerebral lodes metabolism damage. PET was helpful to assess the cognition and metabolism of brain. It was effective means for detecting thecerebral functional impairment of VD.

\section{Time of Hospital Presentation in Patients with Acute Stroke and Other Delay Factors for Thrombolysis}

\author{
Z. Zhang, W. Zhang, O. Bi, G. Zhu \\ Department of Neurology, Beijing General Hospital of \\ PLA, Beijing, China
}

Objective: To supply clinical data for establishing the rescue net before visiting hospital and hospital admission, we have investigated the time of hospital presentation in patients with acute stroke and related delay factors for thrombolysis. Methods: Medical records of 2,914 patients admitted in 35 hospitals in China from Jun 30th 2002 to Apr 30th 2003 were reviewed. We investigated 2,270 patients with complete data. Results: Among them, 621 patients (27.4\%) with onset of symptom were found during daytime, 375 patients in evening, same as the abroad records. Half patients got to hospital from onset place within $3 \mathrm{~h}$. The interval between visiting hospital and performing CT scan was within 30 minutes in 1084 patients $(47.8 \%)$. The interval between performing CT scan and analyzing the findings was within 30 minutes in 221 patients $(53.8 \%)$. 1,250 patients $(55.1 \%)$ were treated within 60 minutes. One hundred and three of $1,778(5.8 \%)$ patients with ischemic strokes underwent thrombolysis. Conclusions: The major factor of time delay before visiting hospital is duration in the way. Asking for emergent help (120/999) may shorten the time delay.

\section{Neuroprotection of Estrogen and it's Molecular Machanism}

\author{
Y. Xu, N. Wang, C. Wang
}

Department of Neurology, Affiliated Drum Tower Hospital, Medical College of Nanjing University, Nanjing, China

Objective: To study neuroprotection of estrogen and it's molecular machanism. Methods: 1 . Estrogen receptor $\alpha(\mathrm{ER} \alpha)$-knockout 
mice with MCAO, wild type mice as control. 2. Primary mice cortex neurons culture with OGD 293 cell lines, which have no estrogen receptor expression for signal research. 3. Estrogen in different dose treated MCAO mice and OGD-neurons that are $25 \mathrm{ug} /$ day (E-25), $50 \mathrm{ug} /$ day (E-50), three days and $100 \mathrm{ul}$ sesame oil as control. 4. Infact was detacted by TTC and neurons death determined by PI/Calcein. 5. Estrogen signals were studied by Estrogen receptors cloning, transfection, gene report assay and immunohistochemistry. Results: 1. Higher dose estrogen (E-50) decrease ischemic infarct with knockout and wild type mice significantly. Lower dose only was usable for wild type. Ischemic infarct(\%) were as following: wild type, $59.6 \pm$ 4.10 (veh), $26.3 \pm 3.9$ (E-25), $22.4 \pm 4.25$ (E-50); knockout mice, $25.6 \pm 3.7$ (veh), $23.5 \pm 4.12$ (E-25), $8.35 \pm 3.47$ (E-50). 2. OGDneurons death was decreased by Estrogen using PI/Calcein (\%), that is $37.07 \pm 3.60$ (veh), $27.5 \pm 3.04(\mathrm{E}-5 \mathrm{nM}), 23.35 \pm 5.01(\mathrm{E}-10 \mathrm{nM})$, $18.82 \pm 3.31(\mathrm{E}-15 \mathrm{nM}), 15.6 \pm 2.85(\mathrm{E}-20 \mathrm{nM}), 25.05 \pm 6.28$ (E-50nM). 3. ER $\alpha$ expressed in neuron, not in 293 cells. $80 \%$ located in nuclear and $20 \%$ in neurites. Estrogen activates ERE function using CAT or Lucifarase assay. GFP- Er $\alpha$ fusions which transfected into 293 cells also have this function. 4. ER $\alpha$ expressed on neurites and colacolizated with MAP Kinase protein using immunohistochemistry, that is membrane-ER activated rapid nongenomic signal pass way and it's important in acute cerebral blood vessel disease. Conclusions: Estrogen activated ER and protected brain and neurons from ischemic or hypoxia injury. ER in nuclear activated ERE genetic pass way and ER in membrane activated MAP Kinase rapid nongenetic pass way.

\section{Blood Pressure and Clinical Outcomes in Acute Ischemic Stroke}

\section{Q. Dong}

Department of Neurology, Huashan Hospital, Fu Dan University, Shanghai, China

Objective: To explore the association of systolic (SBP) and diastolic (DBP) BP during acute ischemic stroke with subsequent clinical outcomes. Methods: The data including 225 patients from the Shanghai Stroke Trial (anticoagulant/control group) with confirmed acute anterior circulation ischemic stroke and fulfillment of follow-up was analyzed. A single casual SBP/DBP was recorded within 48 hours of stroke onset, before any dehydrator was administered. Potential prognostic factors were checked. Clinical outcomes were evaluated: (1) death resulting from any cause within 14 days; (2) death or dependency (BI $0 \sim 55)$ at 3 months. Results: $84 \%(189 / 225)$ of patients had high BP during the acute phase of ischemic stroke, if the JNC-7 definition of hypertension was used. In univariate analysis, hypertension known prior to stroke was associated with high SBP $(P<0.0001)$ and high DBP $(P<0.0001)$; conversely, age was related to low DBP $(P=0.006)$. The relationship between high SBP (OR1.027, $1.001 \sim 1.054, P=0.040$ ), high DBP (OR1.044, $1.002 \sim 1.087, P=0.038$ ) and early death was observed in the unibut not in the multivariate analysis. The lowest frequency of death or dependency at 3 months occurred in patients with a SBP of $141 \sim 150 \mathrm{mmHg}$. And if this level was used as a reference point, both low (OR5.803, $1.440 \sim 23.381, P=0.013)$ and high (OR7.491, $2.074 \sim 27.052, P=0.002)$ SBP increased the risk of poor late outcome. The multivariate regression analysis showed that this U-shaped relationship was independent. No association of DBP during acute stroke and late outcome was seen. Conclusions: High BP was common in acute ischemic stroke. The link between high BP during the acute period and poor early outcome was confirmed, but whether this was independent was unclear. DBP was not associated with late prognosis. However, both high $(\leq 140 \mathrm{mmHg})$ and low $(>150 \mathrm{mmHg})$ SBP were confirmed as independent late prognostic factors.

\section{Serum Level of Homocysteine is Correlated to Carotid Artery Atherosclerosis in Chinese with Ischemic Stroke}

\author{
D. Fan, H. Wang, Y. Shen, H. Zhang, Y. Fu \\ Department of Neurology, Peking University Third \\ Hospital, Beijing, China
}

Objective: To investigate the relationship between serum level of homocysteine (Hcy) and carotid artery atheroscleosis (CAA). Methods: Both sides of the common carotid artery and internal carotid artery in one hundred and twenty-six cases of Chinese patients with ischemic stroke were measured by $\mathrm{B}$-mode ultrasound, which were divided into a normal group and A, B, C, D groups according to the severity of CAAs. With fasting serum, Hcy as well as folate, vitamin B12, and lipids were detected. Results: The mean \pm SD age was $64 \pm 13$ years (range $39 \sim 87$ years). In a logistic regression model, the Hcy concentrations were associated with an elevated risk of CAAs independent of all traditional risk factor. When CAAs became severer, the serum Hcy was higher. The levels of the normal group and A, B, C, D groups were $13.22 \pm 6.15 \mu \mathrm{mol} / \mathrm{L}$, $16.29 \pm 9.81 \mu \mathrm{mol} / \mathrm{L}, 19.49 \pm 11.16 \mu \mathrm{mol} / \mathrm{L}, 27.21 \pm 17.47 \mu \mathrm{mol} / \mathrm{L}$, $24.14 \pm 8.64 \mu \mathrm{mol} / \mathrm{L}$, respectively. Rank test showed a significant difference between control and other groups $(P<0.05)$. The levels of folate and vitamin B12 were negatively correlated with the Hcy concentrations. Spearman correlation coefficient is -0.23 and -0.42 $(P<0.05)$. Conclusions: Hyperhomocysteinaemia is an independent risk factor of CAAs and the degree of CAAs is highly correlated with serum level of Hcy. The causes of hyperhomocysteinemia may be the result from decrease of folate and vitamin B12. Clinical trials are now required to evaluate the effect of treatment with these vitamins in the primary and secondary prevention of cerebral vascular diseases.

\section{Effects of Repetitive Transcranial Magnetic Stimulation on Rats' Motor Cortical Excitability and Neurofunction After Cerebral Ischemia-reperfusion Injury}

\section{Cui, L. Yan, H. Feng \\ Department of Neurology, Peking Union Medical College Hospital, Beijing, China}

Objective: To clarify the effects of repetitive transcranial magnetic stimulation (rTMS) on rats' motor cortical excitability and neurofunction after cerebral ischemia-reperfusion injury. Methods: After determined awake motor threshold (MT) and motor evoked 
potential (MEP) of right hindlimbs, Sprague-Dawley rats were subjected to the middle cerebral artery occlusion and reperfusion injury, then rTMS were applied to them once a day. A week later, MT and MEP were evaluated again, as well as the extent of neurologic deficits graded as different scores using two examinations and infarct volume. The variations of these parameters and the effects of rTMS were analyzed. Results: After cerebral ischemia-reperfusion, both of MT and neurologic deficient scores increased and distinct focal infarction formed. The change of amplitude and latency of MEP was not evident. On the other hand, when compared with control group, the MT and neurological scores of rats receiving rTMS had been improved significantly $(\mathrm{P}=0.02,0.03)$; the infarct volume decreased by $37 \%$, but there is no statistically significance $(P=0.06)$. Conclusions: rTMS may facilitate neurofunction recovery after cerebral ischemia-reperfusion injury, and should be explored further.

\section{Hemodynamics of Brain Arteriovenous Malformation: Clinical Measurement and Theoretical Analysis}

\author{
D. Wang, F. Ling, S. Sun, Z. Yan, Y. Zhang \\ Department of Neurosurgery, Beijing Hospital, \\ Beijing, China
}

Objective: To report the hemodynamic parameter values of brain arteriovenous malformation (AVM) obtainning by clinical measurement and theoretical deduction, and to explore the cause of those changes. Methods: The parameter data including vessel resistance, pressure, flow velocity, flow rate, diameter and vessel wall shear stress at the $A_{1}, M_{1}$ and $P_{1}$ segment respective of the anterior, middle and posterior cerebral artery (ACA-A $\mathrm{A}_{1}, \mathrm{MCA}-\mathrm{M}_{1}$ and PCA- $\mathrm{P}_{1}$ ) were measured or calculated to the brain AVM group (a series of 37 consecutive cases) and normal control group (15 contemporaneous cases without any cardio- and brain vascular disease), all the above data were analyzed statistically and farther deducing or verifying were done with the principle of electricity and minimum work. Results: The statistical analysis on the data of those arteries supplied AVM verse un-supplied or verse normal control indicated that vessel resistance and blood pressure were decreased, flow velocity and flow rate increased, arterial diameter enhanced, but vessel wall shear stress trending conservational, and those changes can be deduce and explain from the formula of electricity and minimum work. Conclusions: The arteriovenous shunt in brain AVM is its low resistance cause in angio-architecture, which leads to low pressure and high flow velocity and flow rate, but the diameter widering and vessel wall shear stress trending conservational in those arteries supplied AVM are the accommodation follows the general principle of minimum work.

\section{Selective Intra-arterial Thrombolysis for Acute Ischemic Stroke}

\author{
S. Wang, J. Wu, D. Wang, J. Feng, S. Fang, M. Rao \\ Interventional Treatment Center for Ischemic Stroke, \\ First Hospital of Norman Bathune College of Medical \\ Sciences, Jilin University, Changchun, China
}

Objective: To explore the best occasio of selective intra-arterial treatment for acute ischemic stroke and analyze the effect factors for curative effect. Methods: Clinical data of 28 cases in Department of Neurology of our hospital during Feb. - Aug. 2004 who suffered from acute ischemic stroke and accepted selective intra-arterial thrombolysis were analyzed retrospectively. There were 19 cases with hypertension, 8 with diabetes mellitus, 14 with cardiac diseases and 1 with polycythemia rubra vera. Results: Arterial agiography results 15 cases accepted angiography in ICA system. Among them, 12 ones showed complete occlusion and 3 ones showed no obvious disorder. After intra-arterial thrombolysis, 10 of the 12 cases had arterial recanalization, three showed serious stenosis and still other 3 showed no obvious disorders. Conclusions: If doctors can choose cases carefully according to indications and operate strictly on interventional standard, intra-arterial thrombolysis is a relatively safe theraputic approach.

\section{Epidemiological Characteristics and Trends of Stroke in China}

\author{
S. Wu, W. Wang \\ Beijing Neurosurgical Institute, Beijing, China
}

Background: Stroke, which became harmful to the lives and health of the middle-aged and aged, is an important issue in the field of public health in China. Surveys showed that the top cause of death was from stroke in urban residents, the second cause of death in rural residents. The mortality rate of stroke increases in recent decade years. Results: Higher incidence rate of stroke in China than in USA and European countries; it was the major cause of death from coronary heart disease (CHD) in USA, but the major cause of death from stroke in China, that was about 4 times higher than in USA. 2. Higher incidence and mortality rates of stroke in northern areas than in Southern areas of China; higher prevalence and incidence rates in urban than rural areas; The mortality rate of stroke was $2 \sim 3$ times higher than that of myocardial infarction (MI). Conclusions: Accordingly, to initiate the campaign of comprehensive prevention and treatment of stroke in community may effectively lower the incidence and mortality rates of cardio-cerebro-vascular diseases. It is an effective and fundamental way for lessening the harm of cardiovascular diseases. 


\section{Different Epidemiological Patterns of VaD and $A D$ in China}

\author{
Z. Zhang, G.E.P. Zahner, G.C. Román, Z. Hong, Q. Qu, \\ X. Liu, X. Zhang, B. Zhou, C. Wu, M. Tang, X. Hong, H. Li \\ Department of Neurology, Peking Union Medical College \\ Hospital, Chinese Academy of Medical Science, Beijing, \\ China
}

Objective: Alzheimer's disease (AD) and Vascular Dementia (VaD) are different diseases, although they share some common risk factors. To better characteristics the epidemiological patterns for AD and $\mathrm{VaD}$, and to better understand entity of $\mathrm{AD}$ and $\mathrm{VaD}$. Methods: Comparison of epidemiological patterns of $\mathrm{AD}$ with $\mathrm{VaD}$ was based on a cross-sectional, door-to-door prevalence survey with a stratified, multi-stage cluster sampling design. Participants were screened with the Chinese Mini-Mental State Examination. Those who screened positive underwent a standardized diagnostic workup. Diagnoses following NINCDS-ADRDA and NINDS-AIREN criteria were confirmed after six months by repeating neuropsychological evaluations. Prevalence odds ratios were estimated in logistic models adjusting for survey design and demographics. Results: We identified 732 AD cases and $295 \mathrm{VaD}$ cases. Prevalence in ages $\geq 65$ was $3.5 \%$ (95\% CI, $3.0 \%-3.9 \%)$ for $\mathrm{AD}$ and $1.1 \%(95 \% \mathrm{CI}, 0.9 \%-1.1 \%)$ for VaD. After post-hoc correction for negative screening errors, prevalence increased to $4.8 \%$ for $\mathrm{AD}$ and remained at $1.1 \%$ for $\mathrm{VaD}$. The different prevalence patterns of $\mathrm{AD}$ and $\mathrm{VaD}$ in China also show in sex- and age-specific trends, geographic distribution, and distribution of ethnicity, education, and marital status. The varied mean age-of-onset, mean duration from onset to diagnosis and mean HIS for AD and VaD are observed. Conclusions: Prevalence patterns of $\mathrm{AD}$ and $\mathrm{VaD}$ in China are different.

\section{Abnormal Ankle Brachial Index and Cardiovascular Disease}

D. $\mathrm{Hu}$

Cardiovascular Deasese Centre, Beijing Tongren Hospital, Beijing, China

Objective: Peripheral arterial disease (PAD) of lower arterial is a clinical condition that often been neglected. PAD is commonly encountered in patients with stroke and coronary artery disease. The population with PAD is at increased risk for cardiovascular morbidity and mortality. This increased risk is observed in both patients with claudication symptoms and those who are asymptomatic. In patients with previous stroke or myocardial infarction, the occurrence of PAD has been shown to increase the risk further. Methods: In the HOPE study, a low ankle brachial index (ABI) index was a strong predictor for cardiovascular mortality and morbidity, suggesting that low ABI has substantial prognostic value. Results: Ramipril reduced the risk of clinical outcomes in those with a clinical evidence of PAD as well as in those without it. However, given that the event rates were higher in those with an $\mathrm{ABI}<0.9$, the absolute benefits are about twice as large in this group (50 per 1,000 events prevented) compared to those with an $\mathrm{ABI}>0.9$ ( 24 per 1,000 events prevented). This suggests that in patients with $\mathrm{CAD}$ and no clinical evidence of PAD, utilizing the $\mathrm{ABI}$ is a simple method for further identify high risk patients who can benefit from preventive strategies. Conclusions: ABI should be used to help define cardiovascular risk in patients with and without symptomatic cardiovascular disease and should be considered in the planning of further large cardiovascular prevention trials.

\section{Initial Experience in Treatment of Crescendo Transient Ischemic Attacks with Endovascular Stent Angioplasty}

\author{
B. Du, W. Jiang \\ Beijing Tiantan Hospital, Capital University of Medical \\ Sciences, Beijing, China
}

Background: Aggressive approachs with urgent surgical or endovascular intervention have been advocated for some of such patients in whom significant extra- or intracranial artery stenosis might be the cause. Purpose: To assess the safety and clinical efficacy of endovascular stenting in patients with extra- and intracranial artery stenosis. Methods: Twenty-one of 364 patients underwent endovascular stenting of symptomatic extra- and intracranial artery stenoses were with a diagnosis of crescendo TIA, including 17 males and 4 females with a mean age of 61 years old. Most of the patients underwent the procedure at least 1 week after treatment with antiplatelet and/or anticoagulant therapy, together with management of vascular risks. Some patients received less than 1 week of medical treatment. Results: The technical successful rate was 95.2\%. One patient did not receive stenting died 1 day after admission, one patient complicated with subarachnoid hemorrhage (SAH) after stenting died 10 days later. For the remaining 19 patients, there were no other periprocedural coplications and no further ischemic attacks during the follow-up period. Conclusions: Endovascular stenting in the treatment of patients with crescendo TIA resulting from extra- and intracranial artery stenoses is safe and effective.

\section{Protein Aggregation After Brain Ischemia \\ B. Hu, M.E. Martone, C. Liu \\ Cerebral Vascular Research Center, Department of Neurology (D4-5), University of Maimi School of Medicine, Miami, USA}

Background: Brain ischemia causes intra-neuronal accumulation of abnormal protein aggregates. Intracellular protein aggregates are highly toxic to the cells. Abnormal protein aggregates are composed of abnormal proteins that are unfolded, misfolded, abnormally modified such as oxidation or phosphorylation, or damaged by free radicals, protease or other enzymes. Overproduction of abnormal proteins in cell causes protein aggregation. Purpose: Protein aggregates containing ubiquitinated proteins are commonly observed in neurons of almost all neurodegenerative disorders, and have been considered as a common cause of neuronal degeneration. Methods: Recently, we have found severe protein aggregation in hippocampal CA1 
neurons after transient forebrain ischemia and in the penumbra neurons after transient focal ischemia. Results: By using ethanolic phosphotungstic acid electron microscopy (EM), ubiquitin immunogold EM, high-resolution confocal microscopy and biochemical analysis, we found that protein aggregates were appeared in the neurons at the early stage after transient ischemia. The neurons would die in a delayed manner, days or weeks after ischemia. The protein aggregates were progressively accumulated in the postischemic neurons until their death. However, these aggregates were virtually absent in the neurons that survive the insult. Protein aggregates appeared as clumps of electron dense materials that stained heavily for ubiquitin and were associated with various intracellular membranous structures. High-resolution confocal microscopy further demonstrated that protein aggregates containing ubiquitin immunoreactivity were persistently and progressively accumulated in the postischemic neurons destined to die, but not in neuronal populations that survive after ischemia. On western blots, ubiquitinated proteins were dramatically increased in the brain regions where neurons would die in the delayed manner during the postischemic phase. The ubiquitinated proteins were Triton-insoluble, indicating that they were irreversibly aggregated. Conclusions: We conclude that proteins are severely damaged ubiquitinated and aggregated in neurons after ischemia. Based on our results, we propose a new hypothesis for ischemic cell death whereby overproduction of unfolded and damaged proteins and irreversible protein aggregation after ischemia ultimately lead to delayed neuronal death.

\section{Brain Injury After Intracerebral Hemorrhage: The Role of Iron}

\section{G. Xi}

Department of Neurosurgery, University of Michigan, Michigan, USA

Background and Purpose: Evidence indicates that brain injury after intracerebral hemorrhage (ICH) is, in part, due to the release of iron from hemoglobin. We, therefore, examined whether such iron is cleared from the brain and the effects of ICH on proteins that may alter iron release or handling: brain heme oxygenase-1, transferrin and transferrin receptor and ferritin. The role of iron in brain edema formation, brain atrophy and neurological deficits was also examined. Methods: Male Sprague-Dawley rats received an infusion of 100$\mathrm{ml}$ autologous whole blood into the right basal ganglia. Enhanced Perl's reaction was used for iron staining and brain non-heme iron content was also determined. Brain heme oxygenase-1, transferrin, transferrin receptor and ferritin were examined by Western blot analysis and immunohistochemistry. Immunofluorescent double labeling was performed to identify which cell types express ferritin. Deferoxamine was used as an iron chelator. Over the period of the experiment, the rats underwent behavioral testing (forelimb placing, forelimb use asymmetry and corner turn tests). Results: ICH upregulated heme oxygenase-1 levels and resulted in iron overload in the brain. A marked increase in brain non-heme iron was not cleared within four weeks. Brain transferrin and transferrin receptor levels were also increased. In addition, brain atrophy in the caudate with prolonged neurological deficits occurred after ICH. Although partial functional recovery happened with time, residual neurological deficits were detectible at 3 months. Deferoxamine reduced brain atrophy and improved behavioral outcomes. Conclusions: ICH results in an accumulation of iron in the brain that is not cleared within three months, which contributes to brain edema formation, brain tissue loss and neurological deficits after ICH. Iron chelation may be a useful therapy for ICH patients.

\section{Neuronal Viability Regulated by Reversible Phosphorylation of Bad}

\author{
J. Krieg/stein \\ Institute for Pharmacology and Toxicology, \\ Philipps-University, Ketzerbach, Germany
}

It is generally accepted that the members of the Bcl-2 family play a crucial role in programmed cell death (apoptosis). We could demonstrate that the expression of Bad was increased tremendously in mouse brain tissue $8 \mathrm{~h}$ after a $30 \mathrm{~min}$ occlusion of the middle cerebral artery. This increase was nearly abolished, when TGF- $\beta 1$ was upregulated in brain tissue by an adenovirus vector containing TGF- $\beta 1$ DNA and, concomitantly, the brain was protected against ischemic damage. An increase in the Bad level and neuronal degeneration was also shown when primary cultures of hippocampal neurons obtained from neonatal rats were treated with staurosporine to cause apoptosis. In addition, TGF- $\beta 1$ inhibited the increase in Bad expression and protected the cells against damage. The neuroprotective effect of TGF- $\beta 1$ seemed to be mediated by phosphorylation of Bad via the MAPK/Erk pathway. Bad is assumed to cause apoptosis by binding to the antiapoptotic Bcl-xl and binds to 14-3-3 proteins. The free Bcl-xl protects now the cells against damage. The question arose whether protein phosphatases dephosphorylate bad and, thereby, cause apoptosis. We demonstrated that the protein phosphatase type 2C (PP2C) dephosphorylated Bad at all phosphorylation sites known. Lipophilic compounds with special structural properties could activate PP2C markedly and increased the apoptotic damage of cultured neurons. Therefore, we suggest PP2C to be involved in neuronal apoptosis.

\section{Primary Central Nervous System Vasculitis \\ J.J. Wang \\ President Wesley Neurology Clinic, Memphis, USA}

The primary central nervous system vasculitis is a granulomatous angiitis in the brain. It is not a rare disorder. However, it is usually missed the first time. Based on the literature and personal experience, the central nervous system vasculitis account for up to $0.5 \%$ of total strokes. Because the symptoms are nonspecific, that makes the diagnosis difficult. The three most common clinical symptoms are altered level of consciousness, headaches, and focal neurological deficit. The diagnostic testing is also difficult to interpret sometimes. CT is basically useless for vasculitis. MRI may give some suggestions, but MRA is not useful. Arterial angiogram is useful if it is medium sized and large vessels. It is sensitive, but nonspecific. Brain biopsy is the most reliable testing. However, the neurosurgeon must be notified to 
get all three matters; gray matter, white matter, and meninges to get positive test. Actually the most sensitive testing is the spinal fluid. More than $90 \%$ of the time it is abnormal, but it is nonspecific. In terms of the treatment, steroids are still the main stay. However, it is a long-term treatment, at least six months ideally two years. If the steroids do not work, immunosuppressant may be added on top of steroids. If the steroids are stopped early, the disease may relapse.

\section{Transient Exposure to HBO Is a Safe and Effective Therapy for Neonates: Experimental Evidences}

\author{
J.H. Zhang \\ Department of Neurosurgery, Loma Linda University \\ Health Science Center, Loma Linda, California, USA
}

Background: Clinical application of HBO to acute cerebral hypoxia-ischemia is retarded partially by the limitation of the therapeutic window that most patients are not available to HBO within the first 3-6 hrs after the onset of stroke. An exception is the young stroke or neonatal hypoxia that a HBO chamber placed in the delivery room could save lives or reduce the neurological deficits of newborns. Methods: we have shown using an established neonatal hypoxia rat pup model that application of HBO (1.5-3 ATA, 100\% $\left.\mathrm{O}_{2}\right)$ for one hr, at one hr after hypoxia insult, preserved tremendously brain weight and morphology up to 6 weeks and improved neurological function. Results: We found no signs of neovascularization, no abnormalities in the structure of the retina and no changes in the protein expression of HIF- $1 \alpha$ and VEGF after hyperoxia exposure. Conclusions: Transient exposure to hyperoxia at hyperbaric pressures prevented brain injury by inhibition of apoptosis. This effective treatment does not cause retinopathy of prematurity.

\section{Intracranial Stenting for Cerebral Atherosclerosis}

\section{R.T. Higashida \\ University of California, San Francisco Medical Center, USA}

Objectives: 1. Discuss the incidence and prevalence of intracranial atherosclerosis. 2. Discuss recent results from treatment of intracranial atherosclerosis by balloon angioplasty and stenting. 3. Discuss potential benefits and risks of interventions for intracranial atherosclerosis between medical therapy, vs. surgery, vs. endovascular therapy. Introduction: A significant cause of ischemic stroke is from intracranial atherosclerotic disease due either to hypoperfusion or distal embolization. The incidence has been reported to be $6 \%-10 \%$ in whites, $6 \%-22 \%$ in blacks, $11 \%$ in hispanics, and $11 \%-22 \%$ in the oriental population. The prognosis and morbidity rate for patients with intracranial stenosis varies widely from $10 \%-46 \%$ per year, independent of medical therapy. The surgical option of an extracranial to intracranial bypass procedure has not been show to be of significant benefit over best medical therapy for these patients. Over the past decade, a number of centers have been reporting upon their experience with intracranial angioplasty and stenting, as a treatment option for patients that have failed maximal medical therapy with anti-platelet and anticoagulant medications. Although prospective randomized studies have not yet been performed, results from these centers have indicated that this procedure is technically feasible and there is good early preliminary data demonstrating efficacy. Technique: A baseline computed tomographic or magnetic resonance brain scan is initially performed to assess for evidence of cerebral ischemia and/or infarction. Hemodynamic quantitative blood flow studies utilizing CT Xenon perfusion imaging, MR perfusion/ diffusion imaging, nuclear medicine perfusion imaging, or positron emission tomography are also performed to assess the degree of perfusion to brain tissue. All patients undergo a four vessel diagnostic cerebral arteriogram to determine the site and degree of stenosis, collateral circulation, and other associated vascular pathology. Patients are then systemically anticoagulated with intravenous heparin ( 100 units $/ \mathrm{kg}$ ), and the lesion is carefully crossed under flouroscopic guidance with a microguidewire (0.014 inches) and a balloon angioplasty catheter $(2.0-4.0 \mathrm{~mm}$ diameter) which matches the normal luminal diameter. The balloon is inflated for 5-10 seconds across the lesion until the plaque is sufficiently dilated. In selected cases, a metallic stent may then be placed across the lesion, to further improve the luminal diameter, and decrease the incidence of vessel dissection with secondary restenosis. Patients are then carefully monitored in the neurological intensive care unit for 24-48 hours, with close attention to anticoagulation levels and blood pressure parameters. They are then discharged on antiplatelet medications, clopidogrel $75 \mathrm{mg} /$ day or ticlopidine $250 \mathrm{mg}$ twice/day for 4-6 weeks, and aspirin $325 \mathrm{mg}$ /day indefinitely. Results: Higashida et al, in 1996, reported upon their early experience with 33 patients treated by intracranial balloon angioplasty and reported a $69.7 \%$ technical success rate with improved neurological outcome, however there was an associated $30.3 \%$ rate of stroke and death, in patients that previously failed best medical therapy. Clark et al reported a series of 17 patients, treating 22 vessels with balloon angioplasty, and reported a success rate of $72 \%$ and a 30 day morbidity rate of $11.7 \%$. Connors et al reported a retrospective analysis of balloon angioplasty in 70 patients with intracranial atherosclerosis. He reported an overall stroke rate of $4.2 \%, 2.9 \%$ mortality, and no technical failures. More recently, several centers have reported upon use of intracranial stents for these lesions. Mori et al, reported upon 10 patients with 12 intracranial atherosclerotic lesions. They reported an $80 \%$ technical success rate in accessing the lesion with a stent, and in those patients that were stented, there were no periprocedural complications and there was significant improvement of neurological symptoms following treatment during the 8-14 months of follow up. Gomez et al also reported a series of 12 patients who underwent elective stenting of the basilar artery after episodes of vertebrobasilar ischemia, in patients who failed medical therapy. Stent placement was successful in all cases, with an improvement in luminal diameter from a mean of $71.4 \%$, to a mean of $10.3 \%$, without any procedural complications. Clinical follow-up at 0.5-16 months (mean 5.9 months), demonstrated no new complications, with clinical improvement in all cases, and only 2 patients with residual symptoms. They concluded that intracranial stenting was feasible, with minimal risk to the patient, however the long term impact was still not known. Discussion: In patients suffering from transient cerebral ischemia, stroke, repetitive strokes, or other focal neurological deficits refractory to best medical therapy, 
from intracranial symptomatic atherosclerotic lesions, intracranial balloon angioplasty and/or stenting may be promising as a useful therapeutic procedure. The development of better balloon catheters, and stent delivery systems, have dramatically decreased the technical difficulties and failures previously associated with this technique. Although best medical therapy has not yet been determined for these patients, extrapolation from the extracranial circulation for carotid atherosclerotic disease, does indicate that if a direct surgical revascularization procedure can be performed within acceptable technical success rates (ie:low periprocedural complication rates), than it may be better than medical therapy in certain types of patients. Clearly, once patients have failed medical therapy with anti-platelet and/or anticoagulant medications, than either an endovascular procedure or surgical bypass procedure may be indicated as a possible therapeutic alternative. Although long term follow up, greater than 2-5 years, is still being collected for patients who have undergone intracranial balloon angioplasty and/or stenting, the short term results appear to be encouraging in terms of improving symptomatic patient outcomes, and decreasing the risk of major stroke. As continued improvements occur both with optimizing medical therapies, and with technical improvements in the endovascular treatment of these patients, there remains continued hope, that better treatment regimens for intracranial atherosclerotic lesions, will be available to treat these patients.

\section{Genes and Gender Alter Outcome from Cardiac Arrest and Cardiopulmonary Resuscitation}

\section{R.J. Traystman, J. Kofler, R. Noppens, P.D. Hurn}

Department of Anesthesiology and Perioperative Medicine, Oregon Health and Science University, Portland, USA

Despite four decades of research concerning cardiac arrest/ cardiopulmonary resuscitation (CPR), clinical outcome remains poor. Much of this research has taken the form of developing new methodological or pharmacological approaches to CPR. Now, however, novel basic science and molecular genetic approaches to CPR are needed to improve our understanding of critical mechanisms of injury during CPR to develop new therapeutic interventions. Poly (ADP-ribose) polymerase (PARP) is an abundant nuclear enzyme, which helps maintain genomic integrity in neurons and other cell types. Inhibition of PARP with pharmacological agents or PARP deficiency via PARP knockout (-/-) animals reduces injury after focal ischemia, but the involvement of PARP after CPR is unknown. We evaluated the effect of CPR in PARP-/- compared to their wild-type controls (SV129). Tissue outcome was analyzed in $\mathrm{H}$ and $\mathrm{E}$ stained brain sections and damage was assessed by calculating the percentage of injured neurons referred to the total number of neurons in an area. We found that the percent of injured neurons was reduced in PARP-/- in all three areas of brain examined: hippocampus (dorsal CA1), posterior caudoputamen and anterior caudoputamen. The \% injured neurons was reduced from wild-type to PARP-/- by $33 \%$ in CA1, $47 \%$ in posterior caudoputamen and $40 \%$ in anterior caudoputamen. We also found that there was a reduction $(35 \%)$ in the $\%$ of injured cells in caudoputamen in animals with upregulated superoxide dismutase (SOD+) compared with their wild-type controls (C57B16). Finally, we examined whether there exists a gender difference in outcome from CPR and found that the $\%$ injured cells are less $(53 \%)$ in normal female than male mice. This gender difference disappeared when the females were ovariectomized, but reappeared when the ovariectomized animals were administered estrogen. These data demonstrate that both genes and gender may be important factors in determining outcome from CPR. Thus genes and gender may represent two new areas for therapeutic targets to alter outcome from CPR.

\section{A New Understanding of the Cell Biology of Ischemic Acidosis}

\section{R. Simon, Z. Xiong}

Robert Stone Dow Neurobiology Laboratories, Portland, USA

$\mathrm{Ca}^{2+}$ toxicity remains the central focus of ischemic brain injury. The mechanism bywhich toxic $\mathrm{Ca}^{2+}$ loading of cells occurs in brain ischemia however has become less clear over the past decade as multiple human trials of glutamate antagonists have failed to show effective neuroprotection in stroke. Acidosis is a common feature of ischemia and is assumed to play a critical role in brain injury, however the mechanism(s) remain ill-defined. A new understanding of the cell biology of ischemic acidosis is that acidosis activates $\mathrm{Ca}^{2+}$ permeable Acid-Sensing Ion Channels (ASICs), inducing glutamate receptor-independent, $\mathrm{Ca}^{2+}$-dependent neuronal injury inhibited by ASIC blockers. Cells lacking endogenous ASICs are resistant to acid injury, while transfection of $\mathrm{Ca}^{2+}$-permeable ASICla establishes sensitivity. In focal ischemia, intracerebroventricular injection of ASIC1a blockers, or knockout of the ASIC1a gene protects the brain from ischemic injury. Thus acidosis injures the brain via membrane receptor based mechanisms with resultant toxicity of $\left[\mathrm{Ca}^{2+}\right] \mathrm{i}$, disclosing new potential therapeutic targets for stroke.

\section{Stent Angioplasty of Symptomatic Carotid Stenoses in High Risk Patients: Is Cerebral Protection Device Necessary?}

\section{S. Husain}

NeuroEndoVascular Therapy Unit, Sir Ganga Ram Hospital and St. Stephens's Hospital, New Delhi, India

Background: Carotid angioplasty and stenting (CAS) is increasingly being used for the treatment of symptomatic and asymptomatic carotid artery disease. Although the North American Symptomatic Carotid Endarterectomy Trial (NASCET) has shown carotid endarterectomy (CEA) to be protective compared with medical therapy alone, its stringent eligibility criteria excluded patients with severe medical, angiographic, and neurological risk factors. We examined the safety and efficacy of stent angioplasty in this high-risk subset for which the perioperative morbidity and mortality of surgery are high. One of the major risks of complication is due to embolization of the clot during the procedure. Various cerebral protection or filter 
devices are available to prevent this complication. The use of additional devices sometimes adds new risk related to these devices and also increases the cost of the procedure. Technical essentials and therapeutic results of carotid stenting without cerebral protection are presented. Materials and Methods: From May 1999 and August 2004, CAS was performed in 96 symptomatic NASCET-ineligible patients. Thirty six $(37.5 \%)$ patients had complete occlusion of contra-lateral carotid. There were 60 males and 36 female, mean ages was 52 years (range 40 to 84 years). Mean lesion length was $22 \pm 7.9 \mathrm{~mm}$, and mean percent stenosis was $85 \pm 8.0 \%$ (reference diameter $5.8 \pm 0.3 \mathrm{~mm}$ ). All the carotids were treated without cerebral protection device. Stents used were Precise self expanding stent (Cordis, Johnson \& Johnson), self expanding Carotid Wallstents (Boston Scientific Inc), Carotid Sinus Stent (Optimed) and Prostar Balloon expandable stent [predilation was performed in 7/35(20\%)]. Followup consisted of serial duplex ultrasonography and clinical assessment in 76 patients at a median of 16 months. Results: On an intention to treat basis, the overall technical success rate for carotid angioplasty was $95 / 96(99 \%)$. Six $(6.2 \%)$ patients had transient focal seizure during balloon inflation, which reversed immediately, uneventfully following balloon deflation. All six patients had complete occlusion of opposite internal carotid artery. Ten patients (10.4\%) had transient bradicardia during balloon inflation which did not require pacing. One patient $(1 \%)$ had a minor stroke $8 \mathrm{hrs}$ after the procedure due to hyperperfusion injury resulting in minimal intracerebral haemorrhage. None of the patient died in 30 days peri-procedure period. Two of $76(2.6 \%)$ patients had TIA involving same arterial territory in follow-up period. Conclusions: Carotid arterial stent placement in a high-risk population has morbidity and mortality rates comparable to those of carotid endarterectomy in a lower risk population. CAS is a safe and viable alternative in such group of patients. Cerebral protection device use probably may not be necessary. However, this issue needs to be addressed in a large randomized trial.

\section{Phosphorylation of bFGF as a Prerequisite for Neuroprotection}

\section{S. Klumpp \\ University of Münster, Germany}

Various growth factors have been shown to possess neuroprotective potency. However, growth factors cannot easily be used for therapy of brain damage because of their limited permeation through the blood-brain barrier. Therefore, we searched for strategies to increase the neuroprotective activity of endogenous growth factors. Here we present evidence that upstream phosphorylation of bFGF itself is crucial for its effect. bFGF phosphorylates in the absence of exogenous kinases. This autophosphorylation is acid-labile, indicating that serine-, threonine- or tyrosine residues are not involved. There are reports in the literature stating that modification of histidine residues inhibits binding of NGF to its receptor and blocks bFGF activity. Therefore, site-directed mutagenesis is performed to unravel the phosphorylation site of bFGF. Importantly, such autophosphorylation of bFGF is a prerequisite for its neuroprotective effect. bFGF treated with alkaline phosphatase is no longer protective, whereas persistently phosphorylated bFGF is neuroprotective, despite the presence of alkaline phosphatase. These results suggest that the inhibition of dephosphorylation of bFGF and probably also of other growth factors could increase their neuroprotective activity.

\section{Acute Ischemic Stroke in Japan: Results of a Nation-wide Survey}

\section{T. Yamaguchi \\ National Cardiovascular Center, Osaka, Japan}

Stroke-related mortality in Japan began to decrease in early 1970 s, and is the third leading cause following total cancer and heart diseases in 2003, although it is still higher than those in the Western countries. This rapid decrease in stroke mortality is mainly attributed to that in hypertensive intracerebral hemorrhage, probably due to improved management of hypertension and changes in nutritional and environmental factors. We carried out a nation-wide hospital survey on acute ischemic stroke in cooperation with 156 hospitals all over Japan from May 1999 to April 2000 (Japan Multicenter Stroke Investigators' Collaboration, J-MUSIC). During the period of one year, 16,922 patients who were consecutively admitted to each participating hospital within 7 days of stroke onset were registered prospectively, and clinical data including baseline characteristics, outcome at discharge and results of neuroradiological examinations were collected at the central office (National Cardiovascular Center) using the common data-sheets. The results of the survey are summarized as follows. Mean age of the subjects was 70.6 years old, and men (68.7 y.o.) were significantly younger than women (73.6 y.o.). Men predominated women with a ratio of 3 to $2(61.3 \%$ vs. $38.7 \%) .43 .4 \%$ of the patients arrived at the hospital using an ambulance service, but $17.0 \%$ of them did by walk. A median score of National Institute of Health Stroke Scale was 5, and its mean score was $8.0( \pm 7.9)$. Patients who visited the hospital $\leq 3$ hours after onset were $36.9 \%$ of the total, and those who came $\leq 6$ hours were $49.5 \%$. But, intravenous thrombolysis was done in only $2.5 \%$, as rt-PA has not been approved in Japan because of several reasons. As a substitute for intravenous rt-PA, intra-arterial superselective application of urokinase (UK) was frequently performed in cardioembolic stroke patients with moderate severity. The effects of intra-arterial UK evaluated by using casecontrol technique in the present study appeared to be favorable, although this treatment has not widely been accepted. Proportion of patients with favorable outcome [modified Rankin scale (mRS) score $\leq 2$ ] was significantly larger in the UK group than in the control group $(50.5 \%$ vs. $34.1 \%, P<0.02$ ). $18.8 \%$ were treated in a stroke (care) unit (SCU) or intensive care unit, but the remainders were managed in general wards. Outcome of acute stroke patients treated in SCU/SU has been reported to be better than those treated in a general ward by European investigators. Although only $17 \%$ were managed in SCU/ICU in the present survey, comparison of outcome between patients treated in SCU/ICU and those in general ward revealed a better outcome in the former, but only in those with NIHSS score $\geq 15$. Among 3 clinical categories of brain infarction, lacunar stroke was the most frequent subtype (36.3\%), followed by atherothrombotic stroke (31.1\%) and cardioembolic stroke (20.4\%). In 2 districts (Kantoh \& Kinki), where large metropolitan cities are included, the proportion of atherothrombotic stroke was larger than that of lacunar stroke. Frequencies of diabetes mellitus and hyperlipidemia as risk factor for stroke were the highest in these districts. These data indicate that life 
style including diet in Japan has gradually been changing (westernization), and that proportion of subtypes of brain infarction are becoming similar to that of Western countries. For evaluation of vascular pathology, MRA was most frequently (60.8\%) applied, followed by carotid ultrasonography $(33.7 \%)$, conventional or digital subtraction angiography (17.1\%). In $16.3 \%$ of the patients, however, vascular evaluation was not performed. To improve the patients' outcome, it is desirable to equip hospitals with SCU/SU, and to treat patients by a well-trained stroke team, including co-medical staffs. Public campaign for educating lay-people to recognize early signs of stroke and the importance of early treatment, contributes to increase the number of patients who have an opportunity of receiving sophisticated treatment and achieving a favorable outcome.

\section{Preconditioning in Brain Ischemia - Promises and Limitations}

\section{T.S. Nowak, Jr., M. Ueda, L. Zhao}

Department of Neurology, University of Tennessee, Memphis, USA

Prior brain insults confer striking protection against subsequent more severe challenges, widely studied in the context of both global and focal ischemia. This presentation will provide an overview of recent results from this laboratory that call into question some of the conventional thinking in the field. Global ischemia studies used ischemic depolarization recording in rat hippocampus during 4-vessel occlusion to provide a highly quantitative definition of optimal preconditioning as well as precise evaluation of insult thresholds for CA1 neuron loss. The key observation is that there are distinct components of transient and persistent protection. Very robust neuron preservation evident one week after a test challenge is attenuated at longer survival times, as evidenced by a regression of the observed depolarization threshold for injury toward that of naïve hippocampus. An approximate doubling of the insult threshold from 4 to $8 \mathrm{~min}$ depolarization does persist at 3 months survival, but longer intervals have yet to be examined. Previous studies in focal stroke models have been interpreted to exclude a role of CBF changes in preconditioning effects, but quantitative autoradiographic $\mathrm{CBF}$ measurements in the Spontaneously Hypertensive Rat directly contradict this view. Brief middle cerebral artery occlusion $(10 \mathrm{~min})$ significantly reduces infarct volume after permanent focal ischemia $24 \mathrm{~h}$ later. However, there remains a precise correlation between the distribution of severe ischemia and tissue injury, with an identical CBF threshold for infarction (approximately $30 \mathrm{ml} / 100 \mathrm{~g} / \mathrm{min}$ ) in naive and preconditioned animals. This indicates that preservation of blood flow is primarily responsible for protection in this model. Together these results emphasize the importance of quantitative assessment of the fundamental variables that impact insult severity in models of ischemic preconditioning.

\section{Medical Treatment of Intracranial Atherosclerosis}

\section{K.S. Wong}

Department of Neurology, Prince of Wales Hospital, Chinese University of Hong Kong, Hong Kong SAR

Intracranial atherosclerosis is the commonest mechanism of stroke in Chinese. The prevalence is about $7 \%$ in normal population aged 40 or above. The prevalence escalates with increasing number of risk factors such as hypertension, diabtetes and hyperlipidemia. Moreover, the presence of intracranial atherosclerotic stenosis greatly increases the risk of subsequent stroke. There are many options for the medical treatment of intracranial atherosclerosis. Potential strategies include: antiplatelet agents, statin, ACEI and aagiotensin receptor blockers. Unfortunately, the WARSS and the WASID study have failed to show that warfarin is useful in patients with intracranial atherosclerosis.

\section{Stroke and Cardiovascular Diseases: a Cardiology Perspective}

\section{Z. LiU}

Division of Cardiovascular Medicine, Ohio State University Medical Center, Columbus, USA

Stroke is one of the leading causes of morbidity and mortality in the world. The social and economical impact to the society is overwhelming. Pathophysiologically, stroke is a presentation of cardiovascular diseases in central nerve system. Many risk factors have been identified for the development of stroke, including age, gender, smoking, diabetes, atherosclerosis, and other cardiovascular diseases. But, hypertension, atrial fibrillation, and congestive heart failure (among other cardiovascular diseases) are by far the most significant ones that are related to the development of stroke. The role of cardiologists in the management of stroke (especially ischemic stroke) has been increasingly recognized over the last decade in the medical community. The cardiologists can help to define the mechanisms of stroke (such as cardiac source for emboli), identify the high risk patient population, initiate important measures for the risk stratification, and develop strategies for stroke prevention (both primary and secondary). Diagnosis and treatment of a variety of cardiovascular diseases especially hypertension and atrial fibrillation are critical parts of stroke prevention and risk stratification. 


\section{Intranasal Delivery of Nerve Growth Factor to Center to Central Nervous System Protecting Against Acute Cerebral Infarction}

\author{
H. Zhao, X. Liu, X. Mao, C. Chen
}

Department of Neurology, Jinling Hospital, Medical

College of Nanjing University, Nanjing, China

Objective: To confirm the reliability and feasibility of intranasal (IN) pathway bypassing the blood-brain barrier (BBB) and demonstrate the potential neuroprotective effects of intranasal nerve growth factor (NGF) on acute cerebral ischemia. Methods: Rats were randomly divided into IN NGF, IV NGF and untreated group $(n=4)$. The concentration of NGF in different brain regions after middle cerebral artery occlusion (MCAO) was measured with ELISA. Rats were randomly assigned to 4 groups: IN vehicle, IN NGF, IV vehicle, IV NGF ( $\mathrm{n}=8$ each). Treatments were initiated $30 \mathrm{~min}$ after the onset of MCAO and then again $24 \mathrm{~h}$ later. Three neurologic behavioral tests were assessed at $24 \mathrm{~h}$ and $48 \mathrm{~h}$. Corrected infarct volumes were determined $48 \mathrm{~h}$ after the onset of MCAO. Results: Olfactory bulb in IN NGF group obtained the highest concentration among all the regions, arriving at $3252 \mathrm{pg} / \mathrm{g}$, followed by hippocampus. The NGF concentrations in olfactory bulb and hippocampus in IN NGF group were significantly higher than that in IV NGF and control group. The infarct volume in IN NGF group was reduced significantly by $38.8 \%$ as compared with that IN vehicle. The vestibulomotor function of IN NGF improved significantly at 24 and $48 \mathrm{~h}$ after the onset of MCAO as compared with that $I N$ vehicle $\left(\mathrm{P}_{24}=0.02\right.$ and $\mathrm{P}_{48}=0.04$, respectively). Conclusions: Intranasal NGF could bypass BBB, reach the $\mathrm{CNS}$, reduce infarct volume and improve neurological function in rats following MCAO. Intranasal delivery of NGF holds a promising treatment for stroke and other CNS disorders.

\section{The Hypoperfusion and Hypometabolism of Language Area may be the Pathogenesy of Aphasia}

\author{
Y. Zhang, Y. Wang, Y. Zhu \\ Department of Neurology, Tiantan Hospital of Capital \\ Medical University, Beijing, China
}

Objective: To approach aphasia pathogenesy by observing cerebral blood flow and metabolism changes of language areas on functional magnetic resomance. Methods: Studied ten cases who suffered from stroke and had aphasia characterstics with the handedness of aphasia battery of China, the western aphasia battery and Frenchy dysarthria battery; not only used CT and MRI to decide the pathological site but also analysed the language areas by magnetic resonance spectroscopy and perfusion weighted imaging. The results compared with that of the same position in the opposite. Results: There were 8 right-handedness and 2 left-handedness in 10 stroke patients, Aphasia types were 5 motor aphasia cases, 4 sensory aphasia cases and 1 conduction aphasia case. MRI suggested that disease sites were at left hemisphere; MRS showed that the language areas aminosaccinic, choline and creatine were reduced than that of the same site of right hemisphere; PWI discovered that the damaged language areas regional cerebral blood volume and regional cerebral blood flow were decreased than that of right hemisphere, Mean transit time and time to peak were increased than that of opposite. Conclusions: Language areas of aphasia patients are hypoperfusion and hypometabolism, which may be the mechanisms of aphasia.

\section{Brain Remodeling and Gliosis after Stroke Treated with Marrow Stromal Cells in the Retired Breeder Rats}

Y. Li, J. Chen, C. Zhang, L. Wang, J. Zhang, M. Katakowski, O. Gao, M. Lu, M. Chopp

Department of Neurology, Henry Ford Health Sciences Center, Detroit, USA

We tested the long-term $(4 \mathrm{~m})$ responses to treatment of stroke in the older adult rat using rat derived bone marrow stromal cells (MSCs). Retired breeder Wistar rats $(10 \sim 12 \mathrm{~m})$ were subjected to middle cerebral artery occlusion (MCAo) alone $(n=5)$, or injected intravenously with $3 \times 10^{6} \mathrm{MSCs}(\mathrm{n}=9)$ at $7 \mathrm{~d}$ after MCAo. A global test was performed to measure the MSC effect on functional recovery from an adhesive-removal patch test and a modified neurological severity score. Bromodeoxyuridine (BrdU), a cell proliferation marker, was injected daily for $14 \mathrm{~d}$ prior to sacrifice $(4 \mathrm{~m})$. Double immunostaining was used to identify cell proliferation and cell types for neuronal axons, astrocytes, oligodendrocytes and microglia. Rats with MSC treatment had significant functional recovery at 1, 2, 3 and $4 \mathrm{~m}$ compared to controls $(P<0.05)$. MSC treatment reduced the infarct volume (liquefaction, $P<0.05$ ) and the thickness of the scar wall $(P<0.05)$ and reduced the numbers of microglia/macrophages within the scar wall $(P<0.01)$. Double staining showed $\mathrm{BrdU}^{+}$preferentially colocalized with astrocytes $\left(\mathrm{GFAP}^{+}\right)$and oligodendrocytes $\left(\mathrm{RIP}^{+}\right)$in the scar boundary zone (SBZ) and in the subventricular zone (SVZ) in the treated rats. Increased expression of an axonal marker $\left(\mathrm{GAP}-43^{+}\right)$, among reactive astrocytes $\left(\mathrm{GFAP}^{+}\right.$, hypertrophy and hyperplasia) was found in the SBZ and the SVZ of the treated rats. Moreover, axonal projections exhibited an overall orientation parallel to elongated processes of reactive astrocytes and toward lesion areas of the MSC treated rats, suggesting that rMSCs may enhance reactive astrocyte related axonal repair in adult brain. In conclusion, MSCs injected at 7 days after stroke improve long-term neurological outcome in older animals. Brain tissue repair is a dynamic ongoing process with reactive gliosis, which persists for at least $4 \mathrm{~m}$ after stroke. Reactive astrocytes responding to MSC treatment of ischemia may also promote axonal regeneration during long-term recovery. 
Basic Fibroblast Growth Factor can Improve
the Treatment Effect of Cerebral Infarction

X. Li, C. Yao

Biopharmaceutical Research \& Development Center of Jinan University, Guangzhou, China

Background and Objective: Basic Fibroblast Growth Factor, bFGF, is neurotrophic factor with multifunctional bioactivity. It can improve vascular formation and microcirculation, accelerate the development and growth of central and peripheral nerve system, and protect the apoptosis of nerve cells. In vitro researches showed that bFGF had potential usefulness as a protection and treatment for stroke. In this study, we would evaluate the therapeutic effectiveness of bFGF on cerebral infarction. Methods: 90 patients with cerebral infarction were divided two groups, control group (40 patients) and bFGF treatment group (50 patients). The ages, disease times, case history and concomitant diseases of two group patients had not significantly difference $(P>0.05)$. The control patients were treated with conventional methods, such as expanding the blood vessel, dissolving throbus, controlling the blood pressure, decreasing the intracranial pressure, reducing the blood viscidity and anti-infective etc. The other patients were treated conventional methods, and in addition with bFGF. 4,000 IU bFGF was injected intramuscularly every day and continuously treated for 20 days. Results: There were 30 and 46 effective cases in the control group and the bFGF treatment group respectively, and the total effective rate was $75 \%$ and $92 \%$ of the control group and the bFGF treatment group respectively $(P<0.05)$. The defect index of nerve function of the control group and the bFGF treatment group was $22.33 \pm 8.31$ and $21.20 \pm 8.96$ respectively before treatment $(P>0.05)$, and it was decreased to $14.38 \pm 8.61$ and $11.10 \pm 6.02$ respectively after treatment $(P<0.05)$. Conclusions: bFGF can improve the therapeutic effectiveness of cerebral infarction with conventional methods, decrease the disability and incidence rate of sequela, and raise patients' life quality.

\section{Angulation of Arteries on the Base of the Brain}

Y. Guan, X. Li, X. Zhou, T. Xiang

Department of Radiology, Huaxi Hospital, Sichuan

University, Chengdu, China

Objective: To investigate the angulation of arteries on the base of the brain and provide the anatomical basis for interventional neuroradiologic procedures. Methods: Vascular casting was made by perfusing acrylonitrile butradiene styrene (ABS) with 25 cases fetal and 36 cases adult cadaveric brains. $\angle \mathrm{C}_{6}-\mathrm{OA}, \angle \mathrm{C}_{7^{-}}$-AChA, $\angle \mathrm{C}_{7^{-}}$ PCoA, $\angle \mathrm{C}_{7}-\mathrm{A}_{1}, \angle \mathrm{C}_{7}-\mathrm{M}_{1}, \angle \mathrm{A}_{1}-\mathrm{ACoA}, \angle \mathrm{PCoA}-\mathrm{P}_{1}, \angle \mathrm{P}_{1}-\mathrm{P}_{2}$, the ramification of $\mathrm{M}_{1}$ segments and the ramification of $\mathrm{BA}$ were measured respectively. Results: Most of the angulations including $\angle \mathrm{C}_{6}-\mathrm{OA}$, $\angle \mathrm{A}_{1}-\mathrm{ACoA}, \mathrm{C}_{7}-\mathrm{PCoA}, \angle \mathrm{PCoA}-\mathrm{P}_{1}, \angle \mathrm{C}_{7}-\mathrm{M}_{1}, \angle \mathrm{P}_{1}-\mathrm{P}_{2}, \angle \mathrm{C}_{7}-\mathrm{AChA}$ (only adult group) and the bifurcation of $\mathrm{BA}$ are obtuse. Most of the angulations including the bifurcation of $\mathrm{M}_{1}, \angle \mathrm{C}_{7}-\mathrm{A}_{1}, \angle \mathrm{C}_{7}-\mathrm{AChA}$ (only fetal group) are acute. A compared study was done between two groups, although significant differences exist in $\angle \mathrm{C}_{7}-\mathrm{A}_{1}, \angle \mathrm{C}_{7}-\mathrm{AChA}$ and $\angle \mathrm{C}_{7}-\mathrm{PCoA}$, the common trait is that the average angle is much bigger in adult group than those in fetal group. The very significant difference exists in $\angle \mathrm{C}_{7}$ - $\mathrm{AChA}$ : It is obtuse angle in one group while it is acute angle in another group. Conclusions: Significant changes exist in the angulation of $\angle \mathrm{C}_{7}-\mathrm{A}_{1}, \angle \mathrm{C}_{7}$-AchA and $\angle \mathrm{C}_{7}$-PCoA accompanying with development. For adult, most of the angulations of arteries on the base of the brain are obtuse and most of the angulations of $\angle \mathrm{C}_{7}-\mathrm{A}_{1}$ and the bifurcation of $\mathrm{M}_{1}$ are acute.

\section{Experimental Research of Mechanism on Oxidative Injury in Treating Cerebral Ischemia Reperfusion Mice with DangguiShaoyaoSan}

\section{Wu, M. Yang, H. Guo}

Diagnostic Department of Chinese Medical Science, College of Traditional Chinese Medicine, Beijing, China

Objective: To observe the function of DangguiShaoyaoSan on free radical injury mouse and verify the curative effect and functional mechanism of DangguiShaoyaoSan. Methods: To reproduce the mouse model and contrast to Ginkgo leaf and Nimodipine. Then, to detect the indexes changes of cerebral tissue, such as contents of Malondialdehyde (MDA) and Glutathione (GSH), the activity of Superoxide Dismutase (SOD) and Glutathione Peroxidase (GSH-Px) in each group mouse on the day 7, 15, and 30 after operation. Results: In the cerebral tissue of model mouse, the activity of SOD, GSH, and GSH-Px decreased obviously, but the contents of MDA increased. DangguiShaoyaoSan could raise the activity of SOD, GSH, and GSH-Px, but lower the contents of MDA, which were super to that of contrast medicine, and submitted weigh-effect relation between low and high dose. Conclusions: DangguiShaoyaoSan can strengthen the capability of anti-oxidation injury, restrain the reaction of lipid peroxidation markedly, and have the notable function of antifree radical injury.

\section{Evaluation of Therapeutic Effects on Carotid Angioplasty and Stenting for Transient Ischemic Attack with Stenosis of Internal Carotid Artery}

G. He, X. Zhang, G. Xi

Department of Neurology, Taihe Hospital Affiliated to Yunyang medical college, Shiyan, China

Objective: To evatuate the safety and therapeutic effects on carotid angioplasty and stenting for transient ischemic attack (TIA) with severity stenosis of internal carotid artery. Methods: 11 cases with severity stenosis of internal carotid artery were underwent percutaneous transluminal angioplasy stening (PTAS). The part of stenosis was expanded by balloon before stent was planted. The protect umbrella were installed in far extremity of stenosis before preexpanding and stent planting in 3 cases with internal carotid stenosis greater 
than $90 \%$ and the balloon postexpanding were underwent when remains stenosis were greater than $40 \%$. The control group included 10 patients with severity internal carotid stenosis were treated by anticoagulate only. Results: After PTAS, the diameter of the part of stenosis were dilated significantly (preoperation $2.79 \pm 1.22 \mathrm{~mm}$, postoperation $5.93 \pm 0.64 \mathrm{~mm}$ ), $P<0.001$. 6 months after operation, none of all patients was attacked TIA or cerebral infarction, nor occured complicating disease and restenosis. But $5(50 \%)$ patients of the control were attacked by TIA and 4 patients (40\%) was onset cerebral infarction. Conclusions: PTAS was one of effective and safety methods for treatment severity carotid artery stenosis.

\section{Correlation of High-sensitivity C-reactive Protein, Fibrinogen and Ultrasonic Index of Carotid Atherosclerosis in Patients with Acute Cerebral Infarction}

\author{
Y. Liang, R. Peng, L. Zhou, Y. Wu, S. Zhao, H. Yao, Y. Zhu \\ Department of Cardiology, Guangzhou Post and Telegraph \\ Hospital, Guangzhou, China
}

Objective: To explore the correlation of high-sensitivity creation protein (Hs-CRP), fibrinogen $(\mathrm{Fg})$ and the ultrasound indexes of carotid atherosclerosis in acute cerebral infarction. Methods: The blood concentration of Hs-CRP and Fg were tested while the carotid arteries were examined by color Dopplle and B-ultrasound in 103 subjects divided into the following groups: acute cerebral infarction group (ACI, $\mathrm{n}=33$, age $67.9 \pm 10.6$ years old), old cerebral infarction group (OCI, $\mathrm{n}=34$, age $69.5 \pm 7.1$ years old), normal controls $(\mathrm{n}=36$, age $65.9 \pm 7.8$ years old). Results: The results showed that the carotid intimal-media thickness (IMT) were significantly increased in all of the diseased groups than those in controls $(P$ all $>0.01)$; The total plaque score (TPS) was thicker in the group of OCI $(8.65 \pm 5.76 \mathrm{~mm})$ than in the group of ACI $(4.68 \pm 2.98 \mathrm{~mm})$ $(P<0.01)$ and also in control $(3.71 \pm 1.35 \mathrm{~mm})(P<0.05)$. The incidence rate of atherosclerous plaque of carotid artery was higher in group of ACI $(72.72 \%)$ than those in group of OCI $(70.59 \%)$ $(P<0.05)$ and control $(16.67 \%)(P<0.05)$. The IMT, TPS and the incidence rate of atherosclerous plaque were no different between in the groups of ACI and OCI. The serum concentration of Hs-CRP in the groups of ACI $(19.54 \pm 37.96 \mathrm{mg} / \mathrm{L})$ was higher than that in the group of OCI $(4.01 \pm 7.22 \mathrm{mg} / \mathrm{L})$ and also in control $(1.66 \pm 1.17 \mathrm{mg} / \mathrm{L})($ all $P<0.01)$; there was no difference in the group OCI and control $(P>0.05)$. The 95\% confidence interval of plasma Hs-CRP was respectively $1.24 \sim 2.08 \mathrm{mg} / \mathrm{L} ; 4.52 \sim$ $34.56 \mathrm{mg} / \mathrm{L} ; 1.4 \sim 6.62 \mathrm{mg} / \mathrm{L}$ in the groups of ACI, OCI and control. The plasma concentration of $\mathrm{Fg}$ was no difference in the three groups $(P>0.05)$. Multiple regression stepwise analysis revealed that the serum concentration of Hs-CRP was positive correlated with the plasma concentration of Fg and TPS; and it was a negative correlation with resistant index. There was negative correlation between the plasma concentration of $\mathrm{Fg}$ and high-density lipoprotein cholesterol. Conclusions: In the acute phase of cerebral infarction, the change in the plasma of Fg was not sensitive. The concentration of Hs-CRP elevated was no correlation with IMT thickened; it was actually correlation with the plasma of $\mathrm{Fg}$ and the total plaque score.

\section{Cerebral Cortex Neuron cDNA Differential Expression Library Construction and Identity of One Novel Gene H3}

H. Ma, W. Liao, T. Chu, Y. Wu, G. Xu, X. Liu

Department of Neurology, Nanjing Jinling Hospital, Nanjing University School of Medicine, Nanjing, China

Objective: We know that the CNS of newborn rats are more easy to regenerate than that of adult ones, especially when accepted fetal spinal cord transplantation. So researchers suspect that if newborn rats with transplantation may express much more regeneration genes. The purpose of the experiment is try to explore the novel regeneration related genes expressed in cerebral cortical neurons of newborn rats during their stage of regeneration after injury, and try to analyze some of them. Methods: In the experiment, we setup the spinal cord hemisection model, transplanted the fetal spinal cord immediately after the injury at the local injured position, constructed the differential expression cDNA library combining the methods of SMART and SSH, BLASTN the EST sequences, in silico cloned one gene from the library and BLASTP the putatively protein, also we verified the existence of the protein using the methods of RT-PCR. Results and Conclusions: We constructed the differential expression cDNA library. After BLASTNed, 25 novel ESTs we submitted to Genbank were enrolled.

\section{Therapeutic Effect of Edaravone on the Patients with Acute Cerebral Infarction}

Q. Di, J. Gu, D. Chen

Department of Neurology, Brain Hospital Affiliated to Nanjing Medical University, Nanjing, China

Objective: Verify the efficacy and the safety of edaravone, a novel free radical scavenger, on acute cerebral infarction (ACI). Methods: We performed a randomized, placebo-controlled, parallel-group, double-blind study on ACI patients. 70 patients, enrolled within $48 \mathrm{~h}$ of onset, were allocated to the edaravone group $(\mathrm{n}=35)$ or the placebo group $(n=35)$ randomly. Edaravone was infused at a dose of $30 \mathrm{mg}$, twice a day, for 14 days. Meanwhile, hydroxyethylrutin was infused at a dose of $200 \mathrm{mg}$ and Aspirin was taken orally at a dose of $50 \mathrm{mg}$ or $75 \mathrm{mg}$, once a day, for 21 days. The therapy of placebo group was similar to that of the Edaravone group except for Edaravone. Thrombolytic agents, anti-thrombin drugs and the other neuroprotective agents were prohibited to use. Before the treatment and at the 7 th-day, the 14th-day and the 21 st-day after the treatment, the neurological deficits and activities of daily living (ADL) were evaluated using European Stroke Scale (ESS) and Barthel Index respectively. At 3 months after onset, all the patients were followed up for survival and ADL. Results: 70 patients were initially enrolled. Of these, 31 were allocated to the edaravone group and 32 to the placebo group for analysis. 7 patients were excluded because of violating the standard of treatment or the study. The age, gender, ESS and ADL before the treatment, past history of two groups are similar. A significant improvement in neurological deficits was observed at 
the 21st-day as evaluated by the increased rate of ESS $(p=0.0006)$, while a significant improvement in ADL was also observed at the 90th-day in the edaravone group $(\mathrm{p}=0.0066)$. Only 2 patient's ALT was increased slightly and one patient had shin rash after the treatment of Edaravone. It may correlate to Edaravone. Conclusions: Edaravone can improve the neurological deficits, impaired activities of daily living, and disability, of ACI patients without serious safety problems. Edaravone is a new effective and safe neuroprotective agent for treating acute ischemic stroke.

\section{Analysis of Risk Factors for First-time Seizures After Initial Cerebrovascular Diseases \\ C. Chen, S. Lang, X. Liu, H. Jia, S. Guo, X. Cheng, T. Chen \\ Neurology Department, PLA General Hospital, Beijing, China}

Objective: The main objective of our study was to assess the risk or predictive factors of early and late epilepsy after first-ever stroke. Methods: In this study, we prospectively and consecutively studied 2,474 patients with initial stroke. Of these, 232 patients had poststroke first-time seizures, 1862 patients had not seizure. Of the 232 epileptic patients, 123 experienced early-onset poststroke seizure, 109 developed late-onset poststroke seizure. All of them were inpatients in the same period after first-ever stroke. We determined twenty-four clinical and radiological indexes, including family history, stroke type, stroke location, lesion size, symptoms or diseases concomitant to epilepsy and lifestyle factors. Odds ratio (OR) and 95\% confidence interval after adjustment for possible confounding variables were calculated by logistic regression. Results: The independent risk factors for early poststroke seizure were large lesion $(\mathrm{OR}=9.36)$, subarachnoid hemorrhage $(\mathrm{OR}=5.28)$, electrolyte disturbance $(\mathrm{OR}=2.10)$, and cortical involvement $(\mathrm{OR}=1.33)$. The independent risk factors for late poststroke seizure were cortical involvement $(\mathrm{OR}=11.84)$ and large lesion $(\mathrm{OR}=1.87)$. In the univariate analysis, hypertension was associated with late seizures $\left(\mathrm{x}^{2}=6.092, P=0.014\right)$, whereas it lost its significance as an independent risk factor for late seizures in the multivariate analysis. Conclusions: The decisive factors of early poststroke epilepsy are large lesion, subarachnoid hemorrhage and cortical involvement. Surprisingly, electrolyte disturbance in stroke patients predicted epilepsy; we explain this finding by cellular biochemical dysfunction after acute stroke. Cortical involvement was the main risk factor of late poststroke epilepsy.

\section{Transcranial Doppler Detection of Microemboli in Chinese Patients with Acute Ischemic Stroke}

\author{
X. Ni, W. Zhu \\ Department of Neurology, Drum Tower Hospital, Medical \\ School of Nanjing University, Nanjing, China
}

Objectives: Transcranial Doppler (TCD) detection of microembolic signal (MES) was used frequently in patients with ischemic stroke. This study was designed to investigate the incidence and clinical significance of cerebral arterial MES in Chinese patients with acute ischemic stroke. Methods: One hundred and fifteen Chinese patients with acute ischemic stroke of middle cerebral artery (MCA) territory were performed bilateral MCA TCD monitoring for $30-60(41.98 \pm 9.03)$ minutes using $2 \times 2 \mathrm{MHz}$ bigated probes within 5 hours to 7 days ( $3.39 \pm 1.52$ days) after onset of symptoms. Results: MES were detected in 5 (4.34\%) of 115 patients. The frequency of MES was $1-5 / \mathrm{h}$. The relative intensity of MES ranged $11 \sim 19(15.6 \pm 2.22) \mathrm{dB}$. MES were found in $1 / 9(11.11 \%)$ of MCA main stem infarcts, $3 / 50(6 \%)$ of MCA small vessel infarcts and 1/33 (3\%) of MCA TIA, respectively. No MES was found in 23 cases with MCA lacunar infarcts. MES was detected more frequently in patients $(5 / 53,9 \%)$ with potential source of emboli than in patients $(0 / 62,0 \%)$ without potential source of emboli. MES was found more frequently in MCA stenosis $(2 / 16,12.5 \%)$, cardiogenic embolism $(3 / 29$, $10.34 \%)$, than in carotid stenosis $(0 / 8,0 \%)$ in this study. Conclusions: The prevalence of MES in Chinese patients with acute ischemic stroke is much lower than that in Caucasian. The main sources of emboli in Chinese patients with acute ischemic stroke are the cerebral artery stenosis and cardiogenic embolism.

\section{Relationship Between Handedness and Language Dominant Hemisphere}

\author{
Y. Zhang, R. Ma, Y. Shen, Y. Zhou, Y. Wang, Y. Wang \\ Department of Neurology, Tiantan Hospital of Capital \\ Medical University, Beijing, China
}

Objectives: To reveal the relationship between handedness and language dominate hemisphere whose mother language are Chinese by handedness assessment and aphasia examination to that hospitalized in our Stroke Unit. Methods: 457 stroke cases whose disease site were one by CT or MRI imaging and falled ill at first time undertook memory and intelligence examination, Ruled out 57 cases who impaired memory or intelligence, the rest cases carried out handedness assessment of aphasia battery of China and the western aphasia battery. Results: Right handedness and no-right handedness were 366, 34 cases respectively among the rest 400 cases; 169 cases had aphasia among 237 left hemispere damaged cases and 9 cases had aphasia amomg 163 right hemispere damaged cases; among 366 right handedness, 158 cases had aphasia among 218 left hemispere damaged cases and 7 cases had aphasia amomg 148 right hemispere damaged cases; among 34 no-right handedness, 11 cases had aphasia among 19 left hemispere damaged cases and 2 cases had aphasia amomg 15 right hemispere damaged cases. 
Conclusions: Many of dextral have left dominant hemispere and few have right dominant hemispere whose mother language is Chinese, so do no-right handedness.

\section{Relationship Between Aphasia Types and Lesion Distribution \\ Y. Zhang, R. Ma, Y. Shen, Y. Zhou, Y. Wang, Y. Wang \\ Department of Neurology, Tiantan Hospital of Capital Medical University, Beijing, China}

Objectives: To reveal relationship between aphasia types and lesion distribution by examing aphasia types and CT, MRI scan to that 98 cases suffered from cerebral infarction and had language disorders hospitalized in our Stroke Unit. Methods: Carried out handedness assessment of aphasia battery of China, the western aphasia battery, grade criterion of the Boston diagnostic aphasia and CT, MRI imaging. Results: 98 cases were all right handedness, Aphasia types were 21 Broca aphasia cases, 15 Wernicke aphasia cases, 2 conduction aphasia cases, 8 transcortical motor aphasia cases, 7 transcortical sensory aphasia cases, 12 transcortical mix aphasia cases, 23 global aphasia cases and 10 anomic aphasia cases. 56 cases were located classical language centers and 38 cases were located other sites; including grade criterion of the boston diagnostic aphasia, $0,1,2,3$, 4 grade had 28,30,14,16, 10 cases respectively and many of 0 and 1 grade were distributed classical language centers. Conclusions: Aphasia types are not all meet with classical language centers; others site damaged also produce aphasia. Lesion site at classical language centers are serious.

\section{Investigation of Neuroprotective Effects of Aspirin Preconditioning and Dose-effect Relationship in Focal Cerebral Ischemia of Rat}

Q. Wang, L. Min

Department of Neurology, First Affiliated Hospital of Jinzhou Medical College, Liaoning, China

Objective: To study the neuroprotective mechanism of aspirin preconditioning in cerebral ischemia injury and dose-effect relationship. Methods: The rats were randomly divided into sham-operative group, cerebral group without aspirin and aspirin preconditioning group. Then the aspirin preconditionting group are divided into 4 groups with different dose aspirin. The aspirin preconditionting groups had been orally given aspirin twice one day for 5 days before establishing MCAO model. Assess the neurological deficent score of rat and measure the activity of NOS in brain tissue after $24 \mathrm{~h}$ focal cerebral ischemia. Results: The two aspirin preconditioning groups with aspirin $5 \mathrm{mg} / \mathrm{kg}$ and $15 \mathrm{mg} / \mathrm{kg}$ not only are better at the neurological deficent score, but their activity of NOS are lower than the cerebral group without aspirin $(P<0.01)$; The other two aspirin preconditioning groups with aspirin $45 \mathrm{mg} / \mathrm{kg}$ and $150 \mathrm{mg} / \mathrm{kg}$ are not different from the cerebral group without aspirin at the neurological deficent score and the activity of NOS $(P>0.05)$. Conclusions: Aspirin in small dose, which has been given prior to the focal cerebral ischemia, plays a neuroprotect role by decreasing the activity of NOS.

\section{The Studies of the Relationship Between Transcient Ischemic Attacks and Platelet GMP-140}

\author{
Y. Zheng, J. Wu, Z. Nie \\ Department Neurology, Second Clinical College, Dalian \\ Medical University, Dalian, China
}

Objective: To study the relationship of transcient ischemic attacks (TIA) and platelet GMP-140. Methods: Eighty-six patients with TIA were selected in hospital. We explore the levels of platelet GMP-140 and TXB2 in plasma. Samples were 86 cases of patients, who were divided into cerebral infarction group and none cerebral infarction group. 30 healthy persons were detected as controls. Results: The levels of GMP-140 and TXB2 in plasma increased significantly in cerebral infarction group as compared with the control group. The levels of GMP-140 in plasma of none cerebral infarction group is higher than the controls. But TXB2 in plasma increases insignificantly. The levels of GMP-140 also increased significantly in cerebral infarction as compared with none cerebral infarction, but TXB2 have insignificantly. Conclusions: Platelet GMP-140 in plasma has important relationship with TIA. The concentrations of GMP-140 in plasma are sensitive index for the activity of platelet. The concentrations of GMP-140 in plasma make a new way to sign the attack of TIA.

\section{Microinvasive Trepanation Plus Injection of Urokinaseinjection through Drainage Tube in the Therapy of Epidural Hematoma}

\author{
L. Zheng, S. Chen, Z. Li \\ Department of Neurosurgery, Jieyang People's Hospital, \\ Jieyang, Guangzhou, China
}

Objective: To investigate microinvasive trepanation plus injection of urokinaseinjection through drainage tube in the therapy of epidural Hematoma and avoid open craniotomy. Selection of cases, Indication and value of distribution were studied. Methods: (1) 76 cases of traumatic subacute epidural hematoma mainly characteristic by midline transition $\leq \mathrm{cm}$, GCS score $8-151 \mathrm{~cm}$, all the cases situation of patients were stabilized. Time of operation was 3-7 days after trauma under local anaesthesia in bedside or local anaesthesia (plus venous anaesthesia in some cases), after trepanation, drainage tube was inserted and injection of certain amount urokinase many times during and after operation. After practice for many years, Indication and selection of cases continuously expande, g Cases complicated with comminuted fracture and epidural hematoma riding over venous sinus, Results: Use this method to treat 76 cases of traumatic epidural hematoms were favourable, no complications were found in 
all the cases. Conclusions: This method is simple, convenient, and economic and with good results, also with little injury, less complication and warmly welcome by the patients and their families, so it has a broad value to spread out.

\section{Experimental Study on the Effects of Composite Salvia Miltiorrhiza on Anti- oxygenation During the Treatment of Thrombolysis in Focus Cerebral Infarction}

L. Min, X. Wang, Y. Yang, X. Liu, L. Yang, W. Ma,

C. Mu, H. Wang

First Affiliated Hospital of Jinzhou Medical College, Department of Nerve Intermal Medicine, Liaoning, China

Objectives: To study the effects of composite salvia miltiorrhiza on anti-oxygenation during the treatment of thrombolysis in focus cerebral infarction. Methods: To make a model of focus cerebra infarction by blockaging middle cerebral artery of rabbit with it's own embolism in artery. After making a successful model, we divided these rabbits randomly into negative contrasting group (A group) thrombolysis in vein with urokinase (B group) and composite salvia miltiorrhiza associated with thrombolysis in vein with urokinase (C group). There were 18 rabbits in each group. All of the three groups were examined by grade of nerve function before and after the experiment and were also detected by oxygen-derived free radicals indications (MDA, SOD and GSH-Px), degree of encephaledema and range of cerebra infarction in 24hours after treatment. Results: (1) Compared with pre-treatment, the degree of nerve function in 3 groups decreased obviously $(P<0.05)$ and it is more obvious in group $\mathrm{C}$ than that in both of A and B. (2) Compared with A group, the content of MDA in group B increased and the activity of SOD and GSH-Px decreased. However, there were no significant difference. By contrast, the content of MDA in group C decreased and activity of SOD and GSH-Px increased. There were statistics difference compared with group A and B (3) It showed the most seriously degree of encephaledema in B while group C the slightest. (4) In 24 hours after treatment, the bulk of average cerebral infarction among groups $\mathrm{A}, \mathrm{B}$ and $C$ is $17.87 \mathrm{~cm}^{3}, 9.82 \mathrm{~cm}^{3}$, and $5.78 \mathrm{~cm}^{3}$. Conclusions: (1) The treatment of thrombolysis in vein with urokinase can decrease the degree of defect of nerve function, reduce the bulk of cerebra infarction and treat effectively focus cerebra infarction. Furthermore, during the treatment of thrombolysis in vein with urokinase in focus cerebra infarction, composite salvia miltiorrhiza showed a promotingeffect. (2) Composite salvia miltiorrhiza can reduce the contain of MDA in cerebra tissue, increase the activity of DOD, GSH-Px and of MDA in cerebra tissue, increase the activity of SOD, GSH-Px and restrain oxygenating.

\section{The Study of Brain SPECT Imaging with ${ }^{99}$ Tc $^{\mathrm{m}}$-HL91 in Patients with Cerebral Infarction

\author{
Y. Wang, S. Liu, X. Li, O. Shen, M. Li, Z. Yan, H. Zhang \\ Department of Neurology, Second Affitiated Hospital, \\ Sun Yat-Sen University, Guangzhou, China
}

Objective: To determine prospectively that the presence and change of hypoxic tissue in different type of patients after cerebral infarction and whether hypoxic tissue may represent the ischemic penumbra. Methods: Patients with hemiplegia and probable hemispheric ischemic stroke were studied using SPECT hypoxic imaging with ${ }^{99} \mathrm{Tc}^{\mathrm{m}}$-HL91 and CT simultaneously within 14 days of onset. Some patients performed ${ }^{99} \mathrm{Tc}^{\mathrm{m}}$-ECD SPECT perfusion imaging or ${ }^{18}$ F-FDG coincidences imaging (glucose metabolism imaging) at same phase. Results: Fifty-seven patients were recruited to the study. Thirty patients sufferring from total or partial anterior circulation infarct showed positive hypoxic imaging (positive rate, $83.3 \%$ or $58.8 \%$ ). Hypoxic tissue was generally distributed in the peripheries of the infarct and more in brain cortex, and showed the shape of dot, piece or strip. 10 of the 30 patients performed ${ }^{99} \mathrm{Tc}^{\mathrm{m}}$-HL91 SPECT again on 7 and 44 days of stroke onest and the hypoxic tissue decreased or disappeared. In 2 patients, hypoxic tissue persisted to the 44th day of onest. Five patients performed perfusion imaging and showed hypoperfusion in cerebral cortex, basal ganglion and even thalamus ipsilateral to infarct. Two of the 5 patients showed positive hypoxic imaging. One patient performed glucose metabolism imaging at 17 days and showed the area of hypometabolism in ischemic hemisphere but obvious tracer activity in the infarct. Conclusions: Hypoxic tissue represents the reversibly damaged tissue after cerebral infarction and can persist to the subacute and chronic phase.

\section{The Effect of Dexamethasone on the Expression of bcl-2 Protein in Adult Rats following Permanent Middle Cerebral Artery Occlusion}

T. Feng, K. Zhu, Y. Wang

Department of Neurology, Beijing Tiantan Hospital, Beijing, China

Objective: To investigate the possible mechanism of the neurotoxic effect of dexamethasone (DEX) on adult rats following focal cerebral ischemia. Methods: The rats models of focal cerebral ischemia were established by permanent middle cerebral artery occlusion (MCA). One hour after ischemia experiment groups were treated with dexamethasone $(0.5 \mathrm{mg} / \mathrm{kg})$ whereas control groups were treated with saline. Immunohistochemistry and picture analyses were used to observe the expression of bcl-2 protein at ipsilateral cerebral hemisphere. Results: The expression of bcl-2 protein at penumbra of control groups began at $3 \mathrm{~h}$, peaked at $24 \mathrm{~h}$, decreased to lower level at $48 \mathrm{~h}$ and $72 \mathrm{~h}$ and returned to baselines at $120 \mathrm{~h}$. Treatment with DEX after ischemia inhibited the expression of bcl-2 protein at penumbra of ischemia from $6 \mathrm{~h}$ to $120 \mathrm{~h}$. Conclusions: Treatment of $\operatorname{DEX}(0.5 \mathrm{mg} / \mathrm{kg})$ following focal ischemia exerts neurotoxic effect 
on adult rats, partially through downregulation of the expression of bcl-2 protein.

\section{Effects of Glucocorticoid Receptor Blockade on the Expression of Caspase-3 following Permanent Focal Cerebral Ischemia in Adult Male Rats}

\author{
T. Feng, Y. Wang, K. Zhu \\ Department of Neurology, Beijing Tiantan Hospital, \\ Beijing, China
}

Objective: To investigate the possible role of glucocorticoid receptor blockade with RU38486 on the apoptosis and the expression of caspase-3 following permanent focal cerebral ischemia in adult male rats. Methods: The experimental groups were treated with RU38486 (20 mg/kg) while the control groups were treated with menstruum. One hour later the rat model of cerebral ischemia were established by permanent left middle cerebral artery occlusion (pMACO). In-suit hybridization and immunohistochemistry were used to study the expressiong of caspase-3. Tunel staining was used to show the change of apoptosis. Results: In control groups TUNEL-positive cell and the expression of caspase- 3 were mainly localized at peripheral ischemia area. The expression of caspase- $3 \mathrm{mRNA}$ and caspase- 3 protein were paraller, beginning at $6 \mathrm{~h}$, peaking at $12 \mathrm{~h}$, remaining at high level from $2 \mathrm{~d}$ to $3 \mathrm{~d}$, be negetive at $5 \mathrm{~d}$. Treatment with RU38486 before ischemia inhibited the expression of caspase- 3 and apoptosis, with the number of positive cell significantly fewer than that of control group from $12 \mathrm{~h}$ to $5 \mathrm{~d}$. Conclusions: Glucocorticoid receptor blockade with RU38486 may inbibited the course of apoptosis after cerebral ischemia, partially due to downregulation of caspase-3. Increasing serum level of glucocorticoid after cerebral ischemia may aggravate the neuron apoptosis.

\section{The Relationship Between Serum Thrombomodulin and Serum TNF- $\alpha$ in Acute Cerebral Infarct with HAP}

\author{
T. Feng, Y. Wang, X. Oi \\ Department of Neurology, Beijing Tiantan Hospital, \\ Beijing, China
}

Objective: To observe the change of thrombomodulin and its relationship with TNF- $\alpha$ in acute cerebral infarct with HAP. Methods: The patients with acute cerebral infarct were divided into the group A $(n=24)$ of pure infarct and the group B $(n=16)$ of acute infarct with HAP, with normal control $(n=13)$. The serum samples were obtained from $1 \mathrm{~d}$ to $21 \mathrm{~d}$ after infarct. The serum level of thrombomodulin and TNF- $\alpha$ were examined by ELISA method and radioimmunoassay respectively. Results: The serum levels of thrombomodulin of the group B were significantly lower than those of the group $\mathrm{A}$ in the first week and $14 \mathrm{~d}$ after infarct $(P \leq 0.05)$, with similar result at $21 \mathrm{~d}$. The serum levels of TNF- $\alpha$ of the group B were significantly higher than those of the group A from the first week to $21 \mathrm{~d}$ after infarct $(P<0.05)$, although HAP had been prohibited in 21d. In the group of acute infarct with HAP the serum levels of thrombomodulin related inversely with the serum levels of TNF- $\alpha$. The relationship between thrombomodulin and TNF- $\alpha$ compromised with Gompertz curve. Conclusions: It's possible that the increase of serum TNF- $\alpha$ in acute infarct with HAP may injury the endotheliocyte of brain vessel and prohibited the anticoagulative system, presenting with downregulation of the level of serum thrombomodulin.

\section{Clinical Analyse of Cerebral Infarction in Young Adults}

\author{
Z. Song, G. Zheng \\ Zhongshan Xiaolian People's Hopital, China
}

Objective: To study the clinic and etiology characteristics of cerebral infarction in young adults. Methods: The etiology, clinical manifestation, focus and so on were analyzed by retrospection method for the young adult patients with cerebral infarction. Results: Dynamic occurring account for $53.3 \%$, atherosclerosis and hypertention account for $53.3 \%$, single focus $71.7 \%$, huge infarction $21.6 \%, 50 \%$ occurred in middle cerebral artery, survival rate $95 \%$. Conclusions: Dynamic occurring and single focus is common. Common etiological causes include atherosclerosis and hypertention. Prognosis is good.

\section{Mechanisms of Conduction Aphasia's Repetition Disturbances}

\author{
Y. Zhang, Y. Wang, Y. Zhu \\ Department of Neurology, Tiantan Hospital of Capital \\ Medical University, Beijing, China
}

Objectives: To approach the mechanisms of conduction aphasia's repetition disturbances by using cognitive psychology methods and to provide theory instructions for rehabilitation trainning of the patients whose language disorder characteristics were repetition disturbances. Methods: Two repetition disturbances men whose language function was normal before suffered from fluent aphasia. Their listening and understanding faculty damaged was not serious while capability of repetition impaired was critical. They were diagnosised conduction aphasia by using the Western Aphasia Battery. The two cases were required to undertake three typical cognitive tests. These tests were phonological comprehensive and recognitive tests (hearing words/pictures matching, similar sounds adjustment), spoken output tests (pseudo-words repetition, pseudo-words reading, ture-words/ pictures naming and ture-words reading) and short-term memory tests, which investigated the cases's capacity of phonological inner processing, phonological production and short-term memory, respectively. We calculated both the correct rates of phonological comprehensive, recognition test and spoken output test and the remembrance span number of short-term memory tests. Results: The two cases normally performed in all comprehensive and recognitive tasks. One case presented the difficulty in all spoken output tasks, in spite of 
keeping intact short-term memory abilities. Others had severe deficits in repeating pseudo-words and short-term memory capacity, although he failed to show cognitive limitation in other phonological output tasks. Conclusions: Repetition deficits of conduction aphasia have some difference mechanisms. The two cases in our study were the impairment at later phonological processing stage and short-term memory capacity, respectively.

\section{Analysis of Correlation Psychological Impact Factors in the Emotion Disorder Induced by Stroke}

\author{
Y. Wang, Y. Wang \\ Department of Neurology, Tiantan Hospital of Capital \\ Medical University, Beijing, China
}

Objective: This study attempted to investigate the relationship between the emotion disorder induced by stroke and the correlation psychological impact factors. Methods: One hundred and seventyseven stroke patients with depression diagnosis according to CCMD-3 criteria were recruited. Hamilton Rating Scale of Depression (HAMD), Hamilton Scale of Anxiety (HAMA), Evaluation Scale of Social Support and Defense Style Questionnaire (DSQ) were used to evaluate the illness severity. Results: There was a correlation between the depression severity and ages and economic status of patients; there was a significant correlation between the depression severity and the defense style and social supports, especially for the middle and elder stroke patients. Conclusions: The results suggested that for middle and elder stroke patients, timely and effectively psychological supports and improving their defense styles will help to prevent and treat the induced emotion disorder.

\section{Cognitive Disorders and Mood Disorders Post Stroke}

Y. Wang

Department of Neurology, First Hospital of Beijing

University, Beijing, China

We should pay more attention to the multiple Cognitive disorders and Mood disorders of the brain after stroke except hemiplegia, such as: Aphasia, agnosia, apracxia, visual-spatial disorder, memory disorder, vascular dementia, anxiety-depression, etc. Above higher dysfunction of the brain also can make the patients disabled and should be evaluated and then we should make out the individuation rehabilitation plan for the patients. The first part states the classifications, localization or criteria of above disorders. The second part reports our study about the classification, ratio, the relationship between the manuality and their linguistic cerebral dominance of Chinese aphasia and compare with the westerners. Among 309 cases with cerebral infarction and cerebral hemorrhage at their acute stage of illness, 139 (44.98\%) with aphasia of 10 types, of whom, 134 cases $(96.40 \%)$ were dextral and $5(3.60 \%)$ were nondextral. 136 patients $(97.84 \%)$ were with aphasia due to lesions of left cerebral hemisphere, of whom,
131 cases $(94.24 \%)$ were dextral and $5(3.60 \%)$ were nondextral. Only 3 dextrals $(2.16 \%)$ were with crossed aphasia due to lesions of right cerebral hemisphere. It was indicated that the mojority of the Han nationality had their linguistic cerebral dominance in left cerebral hemisphere, no matter whether they were dextral, which had no essential difference from Westerner.

\section{TCD Application in Patients with Cerebral Artery Stenosis}

\section{S. Gao}

Department of Neurology, Peaking Union Medical College Hospital, Beijing, China

Criteria for diagnosis of intracranial artery stenosis: Circumscribe increase of blood flow; Waveform of flow pattern with turbulence. The flow velocity will be increased when the degree of stenosis is more than $50 \%$. TCD is able to estimate the degree and length of stenosis probably according to the flow velocity and the flow pattern and sound. Sensitivity for diagnosis of anterior circulation is about $80 \%-90 \%$, specificity is $90 \%-95 \%$ compare with DSA or MRA. TCD is able to diagnose the hemodynamic stenosis of extracranial arteries including common carotid artery, internal carotid artery, external carotid artery, subclavian artery and proximal segment of vertebral artery with $4 \mathrm{MHz}$ probe. TCD is able to detect the collateral supply in patients with severe carotid artery stenosis or occlusion based on the flow direction. The application of TCD in patients with carotid artery stenosis including to estimate the degree of stenosis, the location of occlusion (at proximal segment or intracranial segment distal to OA original), number and type of collateral supply, the stability of plaque (with microembolic signal detection), and the number of co-existing intra- and extracranial artery stenosis. For patients with internal carotid artery stenosis, MES were found more frequency in symptomatic $(10 \%-35 \%)$ than asymptomatic stenosis. Occurrence of MES was associated with severity of stenosis and ulceration of plaque. MES detected with TCD was predicting value for further stroke. For patients with intracranial artery stenosis, the MES were also found more frequency in symptomatic than asymptomatic stenosis and predict the further stroke. Contract to the internal carotid artery stenosis, the site of insonation artery is the origin of MES, the progress of thromboembolism can be detected during MES monitoring. The monitoring probe can be located in unilateral or bilateral temporal window; it will not disturb the surgical area. The flow of middle cerebral artery was recorded continuously. TCD provided the following data: thormboembolism after surgery, the hypoperfusion during clamping and hyperperfursion after surgery. 


\section{Is the Concept 'Vertebrovascular Insufficiency' Still Useful?}

\section{Y. $L i$}

Department of Neurology, The First Shanghai People's

Hospital, Shanghai, China

Vertebrovascular insufficiency (VBI) describes a wide spectrum of clinical entities with a common pathophysiology that is a decreased blood flow through the arteries that supply blood to the base of the brain. This more commonly used term developed in the 1950s after Fisher introduced the term carotid insufficiency to describe TIAs of the anterior circulation, which frequently serve as the prodrome to carotid branch infarcts. Although carotid insufficiency has been dropped from common medical jargon, VBI persists as the term that encompasses all TIA syndromes of the posterior circulation. The brain stem is a focal point of neurologic activity, housing cranial nerves, the reticular activating system, and a series of ascending and descending neurosensory tracts. When this compact area of neurologic activity malfunctions as a result of impaired blood flow, several different but overlapping clinical syndromes can result. Symptoms of VBI include difficulty speaking clearly, nausea and vomiting, numbness, unsteadiness that causes the inability to stand or walk (ataxia), vertigo that begins suddenly, vision changes (such as vision loss, diplopia, or nystagmus), as well as weakness. Vertebrobasilar insufficiency is diagnosed using CT or MRI scans of the brain, and sometimes angiography, or magnetic resonance angiography (MRA). Recent developments in neuroimaging provide new perspectives about the disease's prevalence. Some studies utilizing MRI suggest that $40 \%$ of patients with vertebrobasilar TIAs have evidence of brainstem infarction. So the term of 'vertebrovascular insufficiency' is used less recently and is replaced by the term 'posterior circulation ischaemia'. However, especially the neurologists, family physicians, general internalists, geriatricians, and orthopedists are misusing VBI in China greatly. VBI is commonly diagnosed in adults and the elderly when there is isolate dizziness/vertigo and imaging evidence of cervical spondylosis by the presumption that spondylosis causing insufficient blood supply to ischaemia-sensitive vestibular nuclear resulting dizziness/vertigo (so called vertebrovascular insufficiency, just as its name implies). Most physicians don't pay attention to obtaining detail history and do careful neurological examinations and wrongly believing that isolated dizziness/vertigo in adult and elderly are commonly caused by VBI but not peripheral vestibular disorders and psychiatric disorders. So much the worse, the possible cerebrovascular risk factors of the condition are not being extensively probed (MRI, MRA, CTA, DSA, etc), the condition is considered benign (not TIA or warning of stroke), and the drug treatment of VBI is not evidence-based (control cerebrovascular risk factors, aspirin, or anticoagulants). To thoroughly change the chaotic situation, effective measures should be adopted. First at all, national academic diagnostic criteria should be updated according to the evidences and the misleading term 'vertebrovascular insufficiency' should be replaced by the term 'posterior circulation ischaemia'. Secondly, national wild clinical database should be established. Thirdly, continue medical education programs with newly defined term should be promoted.

\section{The State of Therapying Cerebral Infarction with Snake Venom Preparation within our Country and Abroad}

\author{
J. Yang \\ Research Center of Cerebral thrombosis Diseases, \\ Kunming General Hospital of Chengdu Military Region \\ Kunming, China
}

There hasn't been a specific remedy to treat cerebral infarction at present in the World. Many drugs are using to treat acute cerebral infarction and its restorative stage. Some calcium antagonist and anticoagulant, which used in clinic blood vessel dilation such as heparin calcium; drugs to dissolute thrombosis such as urokinase, streptokinase and rt-PA, which have been researched deeply. But the conclusions were different. The general recognition is that since blood vessel dilation may add cerebral edema, the application of calcium antagonist in acute attack stage has no good effect. While in restorative stage, calcium is blocked into the cell and deposit in the cells, which can prevent the death of cells and improve the function of cerebral cells. The application of anticogulant considered that there is apparent difference with the control group. But its effect to clear the emboli is less than drugs of thrombosis dissolution. It's danger of bleeding also increases the worrying of application. Ultra-early application of thrombosis dissolution remedy can improve the early stage of ischemic waterfall-like reaction, reduce large number yield of the free radical and excitatory amino acid. Early stage thrombosis dissolution promotes blood circulation and reduces the damage of nerve cells. Most scholars think its advantages are more than disadvantages. The problem is that thrombosis dissolution remedy has strict of time limit. In China and even the whole world, few cases have used the remedy within 2-4 hours of disease attack. The bleeding ratio was over $25 \%$ when using streptokinase more than 4 hours of disease attack. Over $20 \%$ when using rt-PA, $6 \%-12 \%$ when using urokinase, the half-life period is shorter (only 15-30 minutes), the recurrent rate caused by the damage of blood vessel endothelial function is $6 \%-12 \%$. Therefore, snake venom preparation has been used to therapy acute cerebral infarction and its restoration stage by Chinese hospitals. 1. The main effective mechanism of snake venom preparation is reducing the fibrinogen and preventing thrombosis further enlarged. 2. Snake venom preparation possesses indirect effect to dissolute thrombosis. Because of lots of FDP produced after fibrinolysis made it can improve the blocked blood vessels passable again. 3. Requirement to time limit is not strict. It is effective to the formed over 8 hours even 24 hours. 4 . For the patients with possible thrombosis caused by hyperplasminemia and high blood viscosity. The snake venom can reduce the fibrinogen and blood viscosity and prevent the thrombosis being formed. 5. It has no good effect to the embolism, which was formed by arterioscherotic plaque scaling and cardiogenic diseases. 6 . Safer: Through the comparison between therapeutic groups and control groups which have been researched in large scale with polycentric, random double blind comparison in our country, increase of cerebral bleeding have not yet been found: From 1979 up to now, hospitals in the main land of China have therapied the cerebral thrombosis diseases with snake venom preparation for 24 years. More than I million cases have been therapied. Most of them were cerebral infarction. China is the county using snake venom preparation the most. Before 1997, there was no double blind random control group research. Since 1997, under the leadership of Health Ministry, the 
Office of Cerebrovascular Diseases organized 2 times (more than 2,000 cases) of double blind random comparative researches and has continued for 6 years. The primitive recognition is that the Defibrase group can reduce defect of Neuro - function, raise vital quality and reduce the recurrence rate while it did not increase the bleeding rate and had no big influence to mortality.

\section{Sleep-related Breathing Disorders and Stroke}

\section{Z. Zhao}

Department of Neurology, Changzheng Hospital, Second Military Medical University, Shanghai, China

Stroke and sleep-related breathing disorders are both common and are associated with significant morbidity and mortality. Several recent large epidemiological studies have shown a strong association between these 2 disorders independent of known risk factors for stroke. Sleep related breathing disorders are composed of habitual snoring, increased upper airway resistance syndrome, periodic breathing, and sleep apnea disorder. In the United States, the prevalence of obstructive sleep apnea disorder has been estimated to be $9 \%$ to $15 \%$ for men and $4 \%$ to $9 \%$ for women between the ages of 30 and 60 years. A meta-analysis of 31 publications with 11,816 patients found a $49 \%$ increase in all types of stroke (ischemic, hemorrhagic, transient ischaemic attack) during the morning hours, as compared with the rest of the day. There are several mechanisms of stroke in sleep-related breathing disorders. The combination of cerebral hypoperfusion and hypercoagulability in sleep apnea disorder may be the underlying pathophysiological mechanism for increased risk of stroke in this population. During and after obstructive apnea episodes, there are sharp swings of arterial blood pressure and cerebral blood flow. The OSA patients' erythrogenin level was higher, and there exist a prothrombotic shift in coagulation balance, with increased factor VII clotting activity, which is a marker of the extrinsic coagulation pathway. Almost 50\% increase in the intima-media thickness, as examined by B-mode Duplex sonography of the common carotid artery (1.429 versus $0.976 \mathrm{~mm}$ ) in OSA individuals as compared with controls without SDB with matched age and comorbid factors. The most common daytime manifestation of SBD is excessive daytime sleepiness, frequently associated with signs of intellectual impairment, poor concentration, memory loss, personality changes, headache and fatigue. During the night, patients may have snoring, cessation of airflow, nocturia or enuresis, automatic behavior; sleep drunkenness (disorientation, confusion upon awakening), hypnagogic hallucinations and night sweats. The rate of seeking medical advice on patients' own initiative is very low, some patients misunderstanding it as senescence. SDB always combined with other diseases, thus make the condition more complex. Physical examination of the upper airways may disclose a deviated nasal septum or swollen turbinates, retrognathia, retrognathia, an enlarged tongue, a hypertrophic uvula, a redundant soft palate, or paralyzed vocal cords. There was a high prevalence of cardiac rhythm disturbances in the night and hypertension in the morning among patients with SBD. The diagnosis need exclude other diseases, such as hypothyroidism, acromegaly, neuromuscular disorders, alcohol, sedative-hypnotics and sleep deprivation. Polysomnography and/or multiple sleep latency test (MSLT) is useful methods to monitor the patients' sleep codition with SDB. Take active treatment to the sleep-related breathing disorders may decrease the morbidity and mortality of cerbral vascular diseases.

\section{Neuroplasticity After Stroke \\ J. Zeng \\ Stroke Center, First Affiliated Hospital, Sun Yat-Sen University, Guangzhou, China}

Background: The death of neural cells in the center of the cerebral infarcts or hematoma after stroke leads to the dysfunction of central nervous system. No effective management is available until now. However, the neurologic dysfunction can be improved spontaneously over time, which is considered to be related to neural plasticity including the plasticity in both structure and function. Structural plasticity means the changes of the expression of synaptophysin (SYN), growth associated protein-43 (GAP-43), Ca2+/calmodulindependent protein kinase II (CaMKII), etc, and the resulting changes of the number of synapse and neuron. Functional plasticity refers to the automatic improvement of neurologic dysfunction involving the compensation of the cortical function of the brain. In addition, atrophy of the thalamencephalon, which presents close connection with the motor-sensory cortex of the brain, may occur secondary to the unilateral cerebral cortical infarction. Supplement of brain derived neural factor (BDNF) can alleviate this secondary pathological change and enhance the neural plasticity. Therefore, the dysfunction after stroke is not only due to ischemic or hemorrhagic lesion, but also related to the whole central nervous system, which may be the underlying reason why the rehabilitation of neural function is so difficult and why the treatment is usually unsatisfied.

\section{Amaurosis Fugax: Clinical and Neuroimaging Analysis}

\author{
Y. Zhang, G. Xu, X. Liu \\ Department of Neurology, Medical College of Nanjing \\ University, Jiangsu, China
}

Background: Amaurosis fugax (AF) is a commonly clinical syndrome caused by internal carotid artery (ICA) stenosis. The epidemiological and clinical data of AF is very rare in Chinese population. Objective: The purpose of this study was to analyze clinical and neuroimaging features of Amaurosis fugax patients in a general hospital in China. Methods: During January 2002 to 2004, February 32 patients were diagnosed with AF. Brain MRI, DSA and TCD were performed in all those patients during their staying in the hospital. Clinical and neruoimaging data were analyzed. Results: Amaurosis fugax was associated with a positive scan in $29.6 \%$ of ICA stenosis. The study population consisted of 26 (81.3) men and 6 (18.7\%) women, with mean age $66.2 \pm 8.6$ years (range, 56-78year). DSA results were as follow: Left-ICA stenosis 5 patients $(15.6 \%)$, bilateral ICA stenosis 15 patients (46.9\%), VA stenosis: 12 patients $(37.5 \%)$. MRI scans 6 months after amaurosis fugax revealed 24 patients 
(75.0\%) had cerebral infarctions, in which, 10 patients $(41.7 \%)$ had multiple cerebral infarction, and 4 patients (16.7\%) had lacunar infarct. Conclusions: Patients with amaurosis fugax have ICA stenosis confirmed by DSA. After the first onset of amaurosis fugax, more than half of the patients developed cerebroinfarctions. These results are in accordance with that found in West countries.

\section{Seizures and Epilepsy following Strokes \\ W. Li, G. Xu, X. Liu \\ Department of Neurology, Jinling Hospital, Medical College of Nanjing University, Jiangsu Province, China}

Background: John Hughling Jackson recognized the relationship between seizures and stroke more than a century ago. Today, stroke has been considered as one of the most important causes of acute symptomatic seizures and epilepsy in the elderly. Purpose: Analysis clinical and epidemiological data concerning the epilepsy patients following stroke in our hospital. Methods: We retrospectively reviewed the epilepsy patients after stroke in a general hospital from 1995 to 2003. Seizure types, onset times, microphysical and neuroimaging results as well as clinical data were analyzed. Results: Seizures occur in about $10 \%$ of stroke patients. Five percent are earlyonset seizures (peak onset within the first day after the stroke) and another 5\% are late-onset seizures (peak onset within 6 to 12 months after the stroke). Epilepsy develops in 3\% to $4 \%$ of the stroke patients (in about one third of the patients with early-onset seizures and about one half of the patients with late-onset seizures). Most seizures are single, either partial or generalized. The most common type of early seizures were generalized seizures, slightly less frequent were simple partial seizures. EEG usually showed focal slow waves or nonspecific slowing of background activity. Different factors appear to predict seizures or PSE. The two most common factors are large infarct size and cortical involvement. Age has not been found to predict seizures or PSE, although some researchers found young age to be a weak predicting factor, but the relative risk of seizures in younger patients compared with older patients did not reach statistical significance. Conclusions: Post stroke early seizures as well as late epilepsy do not significantly affect long-term outcome and rehabilitation of stroke. Management options for early seizures and late epilepsy vary and need to be individualized.

\section{Prognosis Value of Electroencephalogram Combined with Glasgow Score in Stroke Comatose Patients}

\author{
W. Zhu, X. Liu, G. Chen, G. Xu \\ Department of Neurology, Jinling Hospital, Medical \\ College of Nanjing University, Nanjing, China
}

Background: Electroencephalogram (EEG) is an important electrophysiological examination to assess the brain functions of the comatose patients. Glasgow Score is clinically used to evaluate degree of coma. Combining electroencephalogram results with Glasgow Score may be of some value in assessing and predicting the progonisis of comatose patients. Methods: The electroencephalogram and Glasgow Score were evaluated in 69 stroke comatose patients, to explore the correlation between them and the prognosis of these comatose patients. Results: The electroencephalogram suggested that 4 patients with theta waves in EEG were recuperated, 3 patients with electrocerebral silence died. Of the 13 patients showing burst suppression, 8 died, 5 patients improved clinically. Of the 26 patients showing beta activity, 9 died, 17 improved clinically. Of the 23 patients showing delta wave, 1 recuperated, 4 died, and 18 improved clinically. Glasgow Scores of 40 improved patients were $7.21 \pm 0.84$, that of 24 died patients were $4.71 \pm 0.56$, that of 5 recuperated patients were $7.52 \pm 0.91$. Conclusions: Combined with Glasgow Score, electroencephalogram (EEG) could be used to evaluate comparatively the prognosis of the comatose patients.

\section{Cerebral Venous Thrombosis}

J. van Gijn MD FRCP FRCP (Edin)

Utrecht, the Netherlands

Cerebral venous thrombosis may affect one or more cerebral sinuses, cortical veins, or deep veins. The diagnosis is often difficult. Firstly, the clinical features are heterogeneous: headache (sometimes with sudden onset), focal deficits, epileptic seizures or impairment of consciousness, in a variety of combinations and with different degrees of severity. The neurological examination will rarely help to make the diagnosis, except when raised CSF pressure (with thrombosis of the superior sagittal sinus) has caused papilloedema, VIth nerve palsies, or both. Secondly, CT scanning rarely shows direct evidence of sinus thrombosis (dense sinus sign, empty delta sign); more often there are indirect signs in the form of atypical infarction. The unusual aspects are the location (parasagittal regions, thalamus, temporal lobes, lesions in both hemispheres or a combination of supratentorial and infratentorial lesions) and the degree of haemorrhagic infarction (sometimes dense enough to suggest primary intracerebral haemorrhage). For a definitive diagnosis MR scanning is indispensable: this should show non-filling of one or more sinuses as well as evidence of thrombus. The list of causal factors is extensive; most frequent are disorders of coagulation ( $70 \%$ ). In $20 \%$ of patients no contributing factors can be identified. Treatment mostly consists of anticoagulant drugs (coumadin derivatives); randomised trials leave some doubt about the efficacy of this treatment but have at least shown that it is safe. Endovascular treatment by thrombolysis and suction through a transvenous catheter has been performed but not in controlled studies. Sometimes primary causes such as mastoiditis have to be treated separately. The prognosis depends as much on the initial state as on treatment; the proportion with complete recovery ranges from $50-80 \%$ in different case series. Recurrences are reported in at most $10 \%$ of patients. 


\section{The Clinical Analysis of Wallenberg's Syndrome}

H. Zhang, X. Liu, G. Xu

Department of Neurology, Jinling Hospital, Medical

College of Nanjing University, Nanjing, China

Background: Wallenberg's syndrome has a sort of symptoms such as vertigo, numbness and hemiparesis. It is a primary syndrome of medullary diseases. Objective: To study the clinical characteristics of Chinese Wallenberg's syndrome and its changes in brain MRI. Methods: 28 patients' clinical data and changes of brain MRI are collected and analyzed at a general hospital in the past 2 years. Results: In all of 28 cases, 25 cases have such dangerous factors as hypertension, diabetes mellitus, atrial fibrillation and stroke. 21 cases suddenly suffer at motion, in which 1 case suffers after neck manipulation. The chief clinical feature of Wallenberg's syndrome is vertigo, no matter whether vomit, numbness, himiparesis, swallow difficulty, throat pain and ataxia are accompanied. The change of brain MRI is mainly multiple cerebral infarction (21/28, 75\%). Among the 21 cases, there are 10 cases $(10 / 21,47.6 \%)$ of medullary infarction and 5 cases $(5 / 21,23.8 \%)$ of cerebellum infarction. There are 3 cases of medullary hemorrhage, vertebral artery angioma and cerebellopontine schwannoma in the 28 cases. In addition, 5 cases undergo DSA ( 2 cases of posterior inferior cerebellar artery stenosis, 1 case of posterior inferior cerebellar artery occluded, and 1 case of vertebral artery malformation). Conclusions: Wallenberg's syndrome of Chinese is characteristic of vertigo and cerebal infarction in brain MRI, which is similar to foreigners. 